\title{
A Biomimetic Approach to the Rocaglamides Employing Photogeneration of Oxidopyryliums Derived from 3-Hydroxyflavones
}

\author{
Supporting Information \\ Baudouin Gerard, Guilford Jones, II, and John A. Porco, Jr. \\ Department of Chemistry and Center for Chemical Methodology and Library Development, \\ Boston University, 590 Commonwealth Avenue, Boston, MA 02215 (USA) \\ E-mail:porco@chem.bu.edu
}

General Information: ${ }^{1} \mathrm{H}$ NMR spectra were recorded at $400 \mathrm{MHz}$ at ambient temperature with $\mathrm{CDCl}_{3}$ as solvent unless otherwise stated. ${ }^{13} \mathrm{C}$ NMR spectra were recorded at $75.0 \mathrm{MHz}$ at ambient temperature with $\mathrm{CDCl}_{3}$ as solvent unless otherwise stated. Chemical shifts are reported in parts per million relative to $\mathrm{CDCl}_{3}\left({ }^{1} \mathrm{H}, \delta 7.24 ;{ }^{13} \mathrm{C}\right.$, $\delta$ 77.0) or acetone- $\mathrm{d}_{6}\left({ }^{1} \mathrm{H}, \delta 2.04 ;{ }^{13} \mathrm{C}, \delta 207.6,30.0\right)$. Data for ${ }^{1} \mathrm{H}$ NMR are reported as follows: chemical shift, integration, multiplicity $(\mathrm{app}=$ apparent, par obsc $=$ partially obscure, ovrlp = overlapping, $\mathrm{s}=$ singlet, $\mathrm{d}=$ doublet, $\mathrm{t}=$ triplet, $\mathrm{q}=$ quartet, $\mathrm{m}=$ multiplet) and coupling constants. All ${ }^{13} \mathrm{C}$ NMR spectra were recorded with complete proton decoupling. Infrared spectra were recorded on a Nicolet Nexus 670 FT-IR spectrophotometer. Low and high-resolution mass spectra were obtained in the Boston University Mass Spectrometry Laboratory using a Finnegan MAT-90 spectrometer. HPLC analysis was performed on an Agilent 1100 series HPLC (CHIRALCEL OD, Column No. OD00CE-AI015 and Agilent Zorbax SB-C18). Melting points were recorded on a Mel-Temp (Laboratory Devices). Analytical thin layer chromatography was performed using $0.25 \mathrm{~mm}$ silica gel $60-\mathrm{F}$ plates. Flash chromatography was performed using 200-400 mesh silica gel (Scientific Absorbents, Inc.). Yields refer to chromatographically and spectroscopically pure materials, unless otherwise stated. Methylene chloride, acetonitrile, methanol, and benzene were purified by passing through two packed columns of neutral alumina (Glass Contour, Irvine, CA). 3-Hydroxyflavone was purchased from Indofine Chemical Company, Inc. (Hillsborough, NJ). Photochemistry experiments were performed using a Hanovia $450 \mathrm{~W}$ medium pressure mercury lamp housed in a watercooled quartz immersion well or employing an ethylene glycol cooling system (Neslab, RTE-140). Pyrex test tubes (16 x $100 \mathrm{~mm})$ were mounted on a support approximately $0.5 \mathrm{~cm}$ from the immersion well lamp. A uranium filter was obtained from James Glass (Hanover, MA). All other reactions were carried out in ovendried glassware under an argon atmosphere unless otherwise noted. 
Irradiation of 3-hydroxyflavone and methyl cinnamate. To a (16 x $100 \mathrm{~mm})$ test tube was added 3-hydroxyflavone 6 (400 $\mathrm{mg}, 1.7 \mathrm{mmol})$ and methyl cinnamate 13 (650 $\mathrm{mg}, 4$ $\mathrm{mmol}$ ) in $8 \mathrm{~mL}$ of anhydrous acetonitrile. After degassing with argon for $5 \mathrm{~min}$, the mixture was irradiated (Hanovia UV lamp uranium filter, water used for cooling) at $\mathrm{rt}$ for $2 \mathrm{~h}$. The solution was concentrated in vacuo to afford a pink-yellow oil. Purification via flash chromatography (60:40 hexanes/EtOAc) afforded $92 \mathrm{mg}(0.23 \mathrm{mmol}, 15 \%)$ of cyclopenta[b]tetrahydrobenzofuran 14 and $370 \mathrm{mg}(0.94 \mathrm{mmol}, 56 \%)$ of a mixture of cyclopenta[bc]benzopyran 15 and benzo[b]cyclobutapyran-8-one $\mathbf{1 6}$ as colorless solid. 16 was converted to $\mathbf{1 5}$ by thermolysis (EtOAc, $65^{\circ} \mathrm{C}, 4 \mathrm{~h}$ ) to afford exclusively cyclopenta[bc] benzopyran 15.

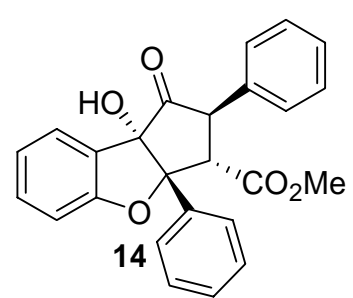

Cyclopenta[b]tetrahydrobenzofuran 14. White solid: $\mathrm{mp}$ 76$78^{\circ} \mathrm{C}$; IR $v_{\max }$ (film): 3449, 3064, 3033, 2955, 2920, 1740, 1697, 1682, 1596, 1476, 1254, 1223, $755 \mathrm{~cm}^{-1}$; ${ }^{1} \mathrm{H}$ NMR (400 MHz, $\left.\mathrm{CDCl}_{3}\right) \delta$ 7.46-6.97 (14 H, m), $4.48(1 \mathrm{H}, \mathrm{d}, J=13 \mathrm{~Hz}), 3.96(1 \mathrm{H}$, d, $J=13 \mathrm{~Hz}), 3.59(3 \mathrm{H}, \mathrm{s}), 3.01(1 \mathrm{H}, \mathrm{s}) \mathrm{ppm} ;{ }^{13} \mathrm{C}$ NMR: 208.9, $168.8,159.6,136.9,134.9,132.1,129.1,129.0,128.9,128.3,134.9$, $132.1,129.1,129.0,128.9,128.3,127.9,126.5,125.8,124.8,122.5$, 110.7, 94.0, 87.8, 59.3, 52.4, $52.3 \mathrm{ppm}$; HRMS (EI) $\mathrm{m} / \mathrm{z}$ calculated for $\mathrm{C}_{25} \mathrm{H}_{20} \mathrm{O}_{5} 400.1311$ found $401.1429(\mathrm{M}+\mathrm{H})$.

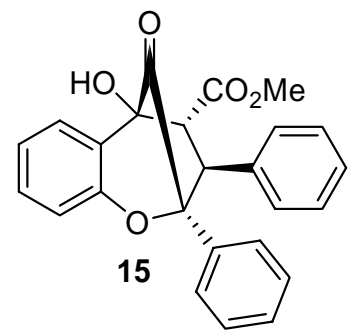

Cyclopenta[bc]benzopyran 15. White solid: $\mathrm{mp} 78-81^{\circ} \mathrm{C}$; IR $v_{\max }$ (film): 3452, 3060, 3033, 2940, 1767, 1736, 1608, 1584, 1483, 1452, 1210, $905 \mathrm{~cm}^{-1} ;{ }^{1} \mathrm{H}$ NMR $\left(400 \mathrm{MHz}, \mathrm{CDCl}_{3}\right) \delta$ 7.34-7.82 (14 $\mathrm{H}, \mathrm{m}), 4.631(1 \mathrm{H}, \mathrm{d}, J=9.2 \mathrm{~Hz}), 3.645(1 \mathrm{H}, \mathrm{d}, J=9.2 \mathrm{~Hz}), 3.606$ $(3 \mathrm{H}, \mathrm{s}), 3.57(1 \mathrm{H}, \mathrm{s}) \mathrm{ppm} ;{ }^{13} \mathrm{C} \mathrm{NMR} \delta 208.4,170.1,150.9,138.2$, $133.4,130.8,129.8,128.9,128.7,128.4,128.0,127.9,127.5,127.4$, $127.3,126.8,126.6,124.9,122.1,116.1,85.1,79.8,57.0,54.2$,

52.8. HRMS $\left(\mathrm{CI} / \mathrm{NH}_{3}\right) \mathrm{m} / \mathrm{z}$ calculated for $\mathrm{C}_{25} \mathrm{H}_{20} \mathrm{O}_{5} 400.1311$ found $401.1357(\mathrm{M}+\mathrm{H})$.

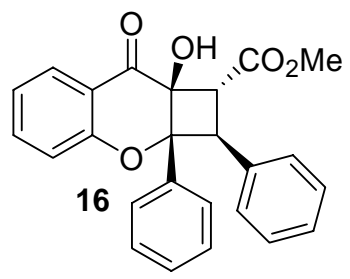

Benzo[b]cyclobutapyran-8-one 16. White solid: $\mathrm{mp} 68-70{ }^{\circ} \mathrm{C}$; IR $v_{\max }$ (film): 3448, 2922, 2851, 1743, 1597, 1558, 1475, 1248, 1055, 998, 965, $755 \mathrm{~cm}^{-1} ;{ }^{1} \mathrm{H} \mathrm{NMR}\left(400 \mathrm{MHz}, \mathrm{CDCl}_{3}\right) \delta$ 7.63-7.61 $(2 \mathrm{H}$, $\mathrm{m}), 7.25-6.95(12 \mathrm{H}, \mathrm{m}), 4.25(1 \mathrm{H}, \mathrm{d}, J=8.8 \mathrm{~Hz}), 3.74(1 \mathrm{H}, \mathrm{d}, J=$ $8.8 \mathrm{~Hz}), 3.55(3 \mathrm{H}, \mathrm{s}), 3.27(1 \mathrm{H}, \mathrm{s}) \mathrm{ppm} ;{ }^{13} \mathrm{C} \mathrm{NMR} \delta 190.33,169.6$, $151.5,139.4,135.4,130.2,129.9,128.9,128.7,128.4,128.1$,

$127.8,127.5,127.4,126.8,124.9,124.6,121.3,116.5,97.5,88.6,60.9,54.3,52.4 \mathrm{HRMS}$ $\left(\mathrm{CI} / \mathrm{NH}_{3}\right) \mathrm{m} / \mathrm{z}$ calculated for $\mathrm{C}_{25} \mathrm{H}_{20} \mathrm{O}_{5} 400.1311$ found $401.1357(\mathrm{M}+\mathrm{H})$. 


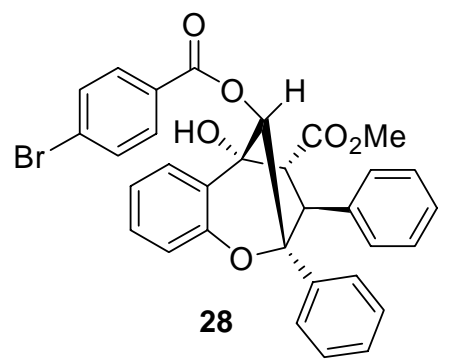

4-Bromobenzoate 28. To a solution of cyclopenta[bc]benzopyran 15 (100 $\mathrm{mg}, 0.25 \mathrm{mmol}, 1$ equiv) in $10 \mathrm{~mL}$ of $\mathrm{MeOH}$ was added sodium borohydride $(15 \mathrm{mg}$, $0.375 \mathrm{mmol}, 1.5$ equiv) portionwise over $5 \mathrm{~min}$ at $0^{\circ} \mathrm{C}$. The resulting solution was warmed to $\mathrm{rt}$ and stirred for $4 \mathrm{~h}$. The reaction was then quenched with sat. $\mathrm{NH}_{4} \mathrm{Cl}$, and diluted with EtOAc $(10 \mathrm{~mL})$ and water $(10 \mathrm{~mL})$. After separation of the organic layer, the aqueous layer was extracted twice with

EtOAc $(5 \mathrm{~mL})$. The organic extracts were combined, washed with brine, dried over $\mathrm{MgSO}_{4}$, filtered, and concentrated in vacuo. The resulting diol $(75 \mathrm{mg}, 0.18 \mathrm{mmol}, 1$ equiv) was directly subjected to acylation using 4-bromobenzoyl chloride (94 $\mathrm{mg}, 0.43$ mmol, 1.2 equiv) and DMAP (44 mg, $0.36 \mathrm{mmol}, 2$ equiv) in $3 \mathrm{~mL}$ of $\mathrm{CH}_{2} \mathrm{Cl}_{2}$. The reaction was stirred at $\mathrm{rt}$ for $24 \mathrm{~h}$. The reaction mixture was diluted using $\mathrm{CH}_{2} \mathrm{Cl}_{2}(5 \mathrm{~mL})$ and washed with water $(2 \times 5 \mathrm{~mL})$. The organic layer was washed with brine, dried over $\mathrm{MgSO}_{4}$, filtered, and concentrated in vacuo. Purification on silica gel $(30 \%$ EtOAc in hexane) provided $95 \mathrm{mg}(0.16 \mathrm{mmol}, 85 \%)$ of 4-bromobenzoate 28 as a colorless solid: mp 73-74 (benzene); IR $v_{\max }$ (film): 3468, 3065, 3032, 2952, 2926, 2854, 1725, 1612, 1590, 1484, 1458, 1269, 911, 754; ${ }^{1} \mathrm{H}$ NMR (400 MHz, $\left.\mathrm{CDCl}_{3}\right) \delta 7.46-7.43(2 \mathrm{H} . \mathrm{d}, J=$ 10.2 Hz), 7.28-7.19 (6 H, m), 7.00-6.90 (10 H, m), 6.47 (1 H, s), 4.20-4.18 (1 H, s, 8.4 $\mathrm{Hz}), 3.80(1 \mathrm{H}, \mathrm{s}), 3.63-3.61(1 \mathrm{H}, \mathrm{d}, J=8.4 \mathrm{~Hz}), 3.48(3 \mathrm{H}, \mathrm{s}) \mathrm{ppm} ;{ }^{13} \mathrm{C} \mathrm{NMR} \delta 170.4$, $166.2,152.0,139.2,136.4,131.7,131.5,129.9,129.2,128.8,128.2,127.9,127.8,127.7$, $126.9,126.5,124.8,123.6,120.9,115.7,87.8,77.8,73.8,60.5,55.3,52.4$; HRMS $\left(\mathrm{CI} / \mathrm{NH}_{3}\right) \mathrm{m} / \mathrm{z}$ calculated for $\mathrm{C}_{32} \mathrm{H}_{25} \mathrm{BrO}_{6} 584.0835$ found $585.0931(\mathrm{M}+\mathrm{H})$.

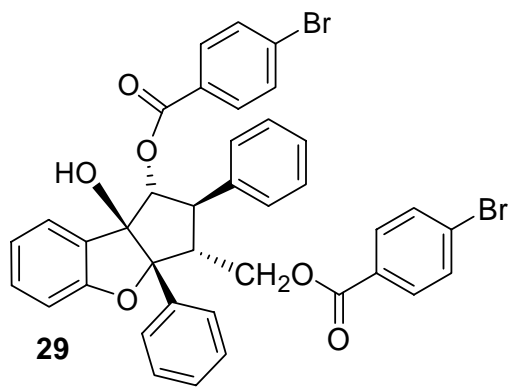

Bis-4-bromobenzoate 29. To a solution of lithium aluminium hydride (26 mg, $0.89 \mathrm{mmol}, 3$ equiv) in THF $(5 \mathrm{~mL})$ at $0^{\circ} \mathrm{C}$ was added a solution of cyclopenta[b]tetrahydrobenzofuran 14 (90 $\mathrm{mg}, \quad 0.225$ mmol, 1 equiv) in $2 \mathrm{~mL}$ of THF. The resulting solution was warmed to $\mathrm{rt}$ and stirred for $3 \mathrm{~h}$. The reaction was then cooled at $0^{\circ} \mathrm{C}$ and quenched with $1 \mathrm{~mL}$ of water followed by $1 \mathrm{~mL}$ of $1 \mathrm{~N}$ aqueous $\mathrm{NaOH}$. The resulting

solution was filtered and the filtrate was evaporated in vacuo to afford the crude triol (63 $\mathrm{mg}, 0.17 \mathrm{mmol}, 75 \%$ ) which was directly subjected to acylation with 4-bromobenzoyl chloride (82 mg, $0.34 \mathrm{mmol}, 2.2$ equiv) and DMAP (63 mg, $0.51 \mathrm{mmol}, 3$ equiv) in $5 \mathrm{~mL}$ of $\mathrm{CH}_{2} \mathrm{Cl}_{2}$. The reaction was then stirred for $24 \mathrm{~h}$ at $\mathrm{rt}$. The mixture was diluted using $\mathrm{CH}_{2} \mathrm{Cl}_{2}(5 \mathrm{~mL})$ and washed with water $(2 \times 5 \mathrm{~mL})$. The organic layer was washed with brine, dried over $\mathrm{MgSO}_{4}$, filtered, and concentrated in vacuo. Purification on silica gel (30\% EtOAc in hexane) afforded $100 \mathrm{mg}(0.14 \mathrm{mmol}, 80 \%)$ of bis-4-bromobenzoate 29 as a colorless solid: $\mathrm{mp} 256-257^{\circ} \mathrm{C}$ (petroleum ether / chloroform); IR $v_{\max }$ (film): 3420 , $3035,2956,2870,1717,1701,1590,1475,1465,1398,1365,1271,1216,1125 \mathrm{~cm}^{-1} ;{ }^{1} \mathrm{H}$ NMR (400 MHz, $\left.\mathrm{CDCl}_{3}\right) \delta$ 7.70-7.68 (2 H, d, J=8.4 Hz), 7.59-7.56 (2 H, d, $\left.J=8.4 \mathrm{~Hz}\right)$, 7.51-7.48 (2 H, d, $J=8.4 \mathrm{~Hz}), 7.40-7.18(14 \mathrm{H}, \mathrm{m}), 6.98-6.59(2 \mathrm{H}, \mathrm{d}, J=8.4 \mathrm{~Hz}), 5.93$ (1 $\mathrm{H}, \mathrm{d}, J=11.2 \mathrm{~Hz}), 4.53(1 \mathrm{H}, \mathrm{dd}, J=11.2,8.4 \mathrm{~Hz}), 4.33(1 \mathrm{H}, \mathrm{dd}, J=11.2,5.6 \mathrm{~Hz}), 3.53$ $(1 \mathrm{H}, \mathrm{m}), 3.19(1 \mathrm{H}, \mathrm{dd}, J=12.4,11.6 \mathrm{~Hz}), 2.98(3 \mathrm{H}, \mathrm{s}) 2.01(1 \mathrm{H}, \mathrm{s}) \mathrm{ppm} ;{ }^{13} \mathrm{C} \mathrm{NMR} \delta$ $166.2,165.4,159.6,137.5,137.0,131.8,131.3,131.2,131.0,129.0,128.7,128.4,128.2$, $127.9,127.8,127.8,127.8,127.7,127.7,126.7,126.5,121.5,110.1,97.5,89.3,86.8$, $62.9,50.4,48.4,29.6 \delta \mathrm{HRMS}\left(\mathrm{CI} / \mathrm{NH}_{3}\right) \mathrm{m} / \mathrm{z}$ calculated for $\mathrm{C}_{38} \mathrm{H}_{28} \mathrm{Br}_{2} \mathrm{O}_{6} 738.0253$ found $739.0217(\mathrm{M}+\mathrm{H})$. 


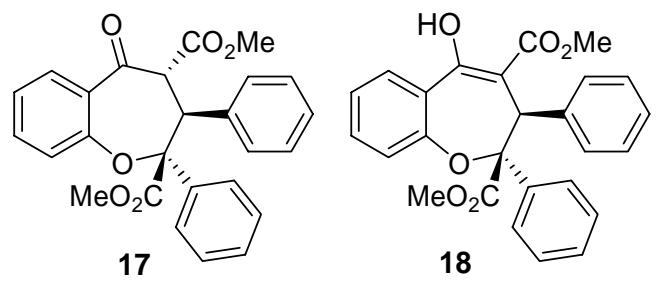

Benzo[b]oxepines 17/18. $50 \quad \mathrm{mg} \quad$ of cyclopenta[bc]benzopyran $15(0.125 \mathrm{mmol}, 1$ equiv) was dissolved in a mixture of methanol $(30 \%)$ and benzene $(0.9 \mathrm{~mL} / 2.1 \mathrm{~mL}) . \mathrm{Pb}(\mathrm{OAc})_{4}$ (55 $\mathrm{mg}, 0.125 \mathrm{mmol}, 1$ equiv) was then added portionwise at $\mathrm{rt}$ and the reaction was stirred for

$30 \mathrm{~min}$ at rt. After removal of the solvent in vacuo, the resulting residue was diluted with water $(5 \mathrm{~mL})$ and EtOAc $(5 \mathrm{~mL})$. After separation of the organic layer, the aqueous layer further extracted twice with EtOAc $(5 \mathrm{~mL})$. The organic extracts were combined, washed with brine, dried over $\mathrm{MgSO}_{4}$, filtered, and concentrated in vacuo. Purification on silica gel (20 \% EtOAc in hexane) afforded $46 \mathrm{mg}(0.11 \mathrm{mmol}, 85 \%)$ of $\mathbf{1 7 / 1 8}$ as a colorless solid (2:1 mixture of keto/enol tautomers by ${ }^{1} \mathrm{H}$ NMR): mp $178-181^{\circ} \mathrm{C}$; IR $v_{\max }$ (film): $3060,3033,2959,2924,1759,1747,1684,1602,1444,1434,1308,1244 ; 1102 \mathrm{~cm}^{-1}$; $\operatorname{NMR}\left(400 \mathrm{MHz}, \mathrm{CDCl}_{3}\right) \delta$ 7.64-7.62 $(2 \mathrm{H}, \mathrm{d}, J=7.2 \mathrm{~Hz}), 7.44-7.28(8 \mathrm{H}, \mathrm{m}), 7.18-7.16$ $(4 \mathrm{H}, \mathrm{m}), 5.12(1 \mathrm{H}, \mathrm{d}, J=10 \mathrm{~Hz}), 4.41(1 \mathrm{H}, \mathrm{d}, J=10 \mathrm{~Hz}), 3.66(3 \mathrm{H}, \mathrm{s}), 3.16(3 \mathrm{H}, \mathrm{s})$ ppm; ${ }^{13} \mathrm{C}$ NMR $\delta 193.2,156.7,154.2,139.0,134.8,132.4,129.2,129.1,128.9,128.7$, 128.3, 128.2, 127.8, 127.7, 127.6, 127.4, 126.9, 126.7, 122.3, 121.9, 121.8, 121.6, 64.9, $52.5,52.2,52.0,51.8,49.8,46.7$. $\delta$ HRMS $\left(\mathrm{CI} / \mathrm{NH}_{3}\right) \mathrm{m} / \mathrm{z}$ calculated for $\mathrm{C}_{26} \mathrm{H}_{22} \mathrm{O}_{6}$ 430.1416 found $431.1516(\mathrm{M}+\mathrm{H})$.

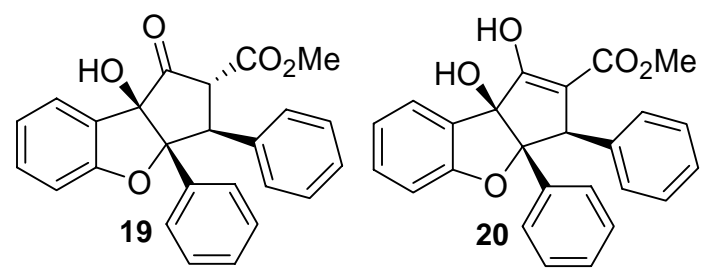

Cyclopenta[b]tetrahydrobenzofurans $19 / 20$. To a solution of cyclopenta[bc] benzopyran $\mathbf{1 5}$ (50 mg, $0.125 \mathrm{mmol}, 1$ equiv) in $\mathrm{MeOH}$ (3 $\mathrm{mL})$ at $\mathrm{rt}$ was added a solution of $\mathrm{NaOMe}(17$ $\mathrm{mg}, 0.31 \mathrm{mmol}, 2.5$ equiv) in $\mathrm{MeOH}(1 \mathrm{~mL})$ at rt. The resulting solution was stirred for 40 $\min$ at $65^{\circ} \mathrm{C}$. After quenching the reaction with sat. $\mathrm{NH}_{4} \mathrm{Cl}$ at rt, $10 \mathrm{~mL}$ of EtOAc was added. The organic layer was separated and washed with water $(2 \times 5 \mathrm{~mL})$ and brine $(5 \mathrm{~mL})$, dried over $\mathrm{MgSO}_{4}$, filtered, and concentrated in vacuo. Purification on silica gel (20\% EtOAc in hexane) afforded $45 \mathrm{mg}$ $(0.11 \mathrm{mmol}, 90 \%)$ of the corresponding rocaglates $\mathbf{1 9} / \mathbf{2 0}$ as a white solid: $\mathrm{mp} 141-143^{\circ} \mathrm{C}$ IR $v_{\max }$ (film): 3066, 3027, 2954, 2923, 2856, 1758, 1730, 1650, 1594, 1454, 1279, 1247, $1146,975 \mathrm{~cm}^{-1} ;{ }^{1} \mathrm{H}$ NMR $\left(400 \mathrm{MHz}, \mathrm{CDCl}_{3}, 1: 1\right.$ mixture of keto/enol tautomers 19:20) $\delta$ 7.52-6.88 (28 H, m), $5.28(1 \mathrm{H}, \mathrm{s}), 4.13(2 \mathrm{H}, \mathrm{dd}, J=13.6 \mathrm{~Hz}), 3.63(3 \mathrm{H}, \mathrm{s}), 3.57(3 \mathrm{H}$, s), $2.66(1 \mathrm{H}, \mathrm{s}), 2.10(1 \mathrm{H}, \mathrm{s}) \mathrm{ppm} ;{ }^{13} \mathrm{C}$ NMR $\delta 204.3,167.1,159.8,132.6,131.1,128.8$, $128.0,127.8,127.7,127.6,127.6,127.3,127.2,126.9,126.8,126.6,126.2,125.3,124.8$, $122.6,121.8,119.5,112.4,110.6,98.7,57.4,56.7,55.8,52.9,51.7$ ppm. HRMS (EI) m/z calculated for $\mathrm{C}_{25} \mathrm{H}_{20} \mathrm{O}_{5} 400.1311$ found $401.1427(\mathrm{M}+\mathrm{H})$. 


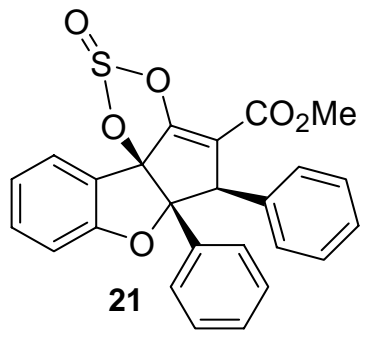

1,3,2-Dioxathiolane 21. To a solution of $\mathrm{NaH}$ (washed with $3 \times 10$ $\mathrm{mL}$ hexanes, $5 \mathrm{mg}, 0.21 \mathrm{mmol}, 2.1$ equiv) in THF $(2 \mathrm{~mL})$ was added a solution of cyclopenta[bc]benzopyran 15 (40 mg, 0.10 mmol, 1 equiv) in THF $(1 \mathrm{~mL})$ at $\mathrm{rt}$. The resulting yellow solution was stirred at rt for $30 \mathrm{~min}$. After addition of thionyl chloride (15 $\mu \mathrm{L}, 0.21 \mathrm{mmol}, 2.1$ equiv) at $\mathrm{rt}$, the mixture was stirred for a further $1 \mathrm{~h}$ and then quenched with sat. aqueous $\mathrm{NaHCO}_{3}$.

$10 \mathrm{~mL}$ of EtOAc was added and the organic layer was washed with $2 \times 3 \mathrm{~mL}$ of water and $3 \mathrm{~mL}$ brine. The organic extracts were dried over $\mathrm{MgSO}_{4}$, filtered, and concentrated in vacuo. Purification on silica gel (5\% EtOAc in hexane) afforded $21 \mathrm{mg}(0.048 \mathrm{mmol}$, $48 \%$ ) of the corresponding 1,3,2-dioxathiolane $\mathbf{2 1}$ as a yellow oil. IR $v_{\max }$ (film): 3025 , 2948, 2913, 1716, 1650, 1553, 1243, 1200, $746 \mathrm{~cm}^{-1} .{ }^{1} \mathrm{H}$ NMR $\left(400 \mathrm{MHz}, \mathrm{CDCl}_{3}\right) \delta 7$. 46-7.07 (14 H, m), $3.85(1 \mathrm{H}, \mathrm{s}), 3.72(3 \mathrm{H}, \mathrm{s}) \mathrm{ppm} ;{ }^{13} \mathrm{C}$ NMR $\delta 190.4,165.4,144.9$, $143.1,132.9,132.6,130.8,130.3,130.0,129.6,129.2,128.8,128.7,128.5,128.1,125.6$, 124.7, 122.6, 111.1, 52.6, 52.4; HRMS (EI) $\mathrm{m} / \mathrm{z}$ calculated for $\mathrm{C}_{25} \mathrm{H}_{18} \mathrm{O}_{6} \mathrm{~S} 446.0824$ found $447.0805(\mathrm{M}+\mathrm{H})$.

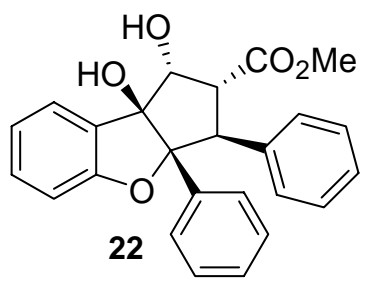

Cyclopenta[b]tetrahydrobenzofuran 22. To a solution of $197 \mathrm{mg}$ $\left(0.75 \mathrm{mmol}, 6\right.$ equiv) of $\mathrm{Me}_{4} \mathrm{NBH}(\mathrm{OAc})_{3}$ and $68 \mu \mathrm{L}(1.25 \mathrm{mmol}$, 10 equiv) of acetic acid in $3 \mathrm{~mL}$ of $\mathrm{CH}_{3} \mathrm{CN}$ was added a solution of $50 \mathrm{mg}$ ( $0.12 \mathrm{mmol}, 1$ equiv) of keto rocaglate $\mathbf{1 9 / 2 0}$ in $1 \mathrm{~mL}$ of $\mathrm{CH}_{3} \mathrm{CN}$. The resulting yellow solution was stirred for $12 \mathrm{~h}$ at $\mathrm{rt}$ before being quenched with $2 \mathrm{~mL}$ of sat. $\mathrm{NH}_{4} \mathrm{Cl}$ solution. The

solution was then treated with $1 \mathrm{~mL}$ of a $3 \mathrm{M}$ aqueous solution of sodium/potassium tartrate and stirred at $\mathrm{rt}$ for $30 \mathrm{~min}$. The aqueous solution was extracted with $\mathrm{CH}_{2} \mathrm{Cl}_{2}(2 \mathrm{x}$ $5 \mathrm{~mL}$ ). The combined organic layers were washed with brine, dried over $\mathrm{MgSO}_{4}$, filtered, and concentrated in vacuo. Purification on silica gel (20\% EtOAc in hexane) afforded 30 mg $(0.047$ mmol, $95 \%)$ of 22 as a white solid: $\mathrm{mp} 176-178^{\circ} \mathrm{C}$; IR $v_{\max }$ (film): 3421 , 3031, 2925, 1733, 1600, 1476, 1462, 1249, 1102, $976 \mathrm{~cm}^{-1}$; ${ }^{1} \mathrm{H}$ NMR (400 MHz, $\left.\mathrm{CDCl}_{3}\right)$ $\delta$ 7.41-6.96 (14 H, m), $4.84(1 \mathrm{H}, \mathrm{d}, J=6 \mathrm{~Hz}), 4.50(1 \mathrm{H}, \mathrm{d}, \mathrm{J}=13.6 \mathrm{~Hz}), 3.99(1 \mathrm{H}, \mathrm{dd}$, $J=6,13.6 \mathrm{~Hz}), 3.66(3 \mathrm{H}, \mathrm{s}),, 2.55(1 \mathrm{H}, \mathrm{s}), 1.82(1 \mathrm{H}, \mathrm{s}), \mathrm{ppm} ;{ }^{13} \mathrm{C}$ NMR $\delta 171.5,159.1$, 136.8, 134.5, 131.4, 127.9, 127.7, 127.6, 127.5, 127.4, 126.8, 126.5, 126.3, 121.6, 111.0, $100.8,93.3,79.2,56.0,52.2,50.8$ ppm LRMS (ESI + ) $\mathrm{m} / \mathrm{z}$ calculated for $\mathrm{C}_{25} \mathrm{H}_{22} \mathrm{O}_{5}$ 402.1467 found $403.0(\mathrm{M}+\mathrm{H})$.

Irradiation of 3-hydroxy-5,7-dimethoxyflavone and methyl cinnamate. To a 16 x 100 $\mathrm{mm}$ test tube was added with kaempferol derivative $7(200 \mathrm{mg}, 0.61 \mathrm{mmol})$, methyl cinnamate 13 (990 mg, $6.1 \mathrm{mmol})$, and $20 \mathrm{~mL}$ of anhydrous methanol. After degassing with argon, the mixture was irradiated (Hanovia UV lamp, uranium filter) at $0^{\circ} \mathrm{C}$ for $12 \mathrm{~h}$ under an argon atmosphere. The solution was concentrated in vacuo to afford a yellow oil. Purification via flash chromatography (60:40 hexanes/EtOAc) afforded $100 \mathrm{mg}(0.2$ mmol, $33 \%$ ) of the corresponding trimethoxy cyclopenta[bc]benzopyran derivative $\mathbf{2 3}$ (mixture of endo/exo cycloadducts) as a white solid and $50 \mathrm{mg}(0.1 \mathrm{mmol}, 17 \%)$ of benzo[b]cyclobutapyran-8-one derivative $\mathbf{2 4}$ as a yellow solid. 


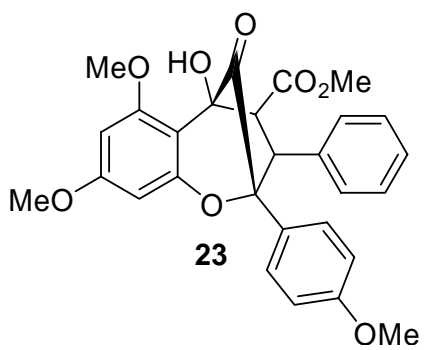

Trimethoxy cyclopenta[bc]benzopyran 23. White solid: mp $83-85^{\circ} \mathrm{C}$. IR $v_{\max }$ (film): 3475, 3013, 2943, 2832, 1786, 1737, $1611,1590,1510,1450,1255,1146,1094,828 \mathrm{~cm}^{-1} ;{ }^{1} \mathrm{H} \mathrm{MR}$ $\left(400 \mathrm{MHz}, \mathrm{CDCl}_{3}\right) \delta 7.54-7.52(2 \mathrm{H}, \mathrm{d}, J=8.8 \mathrm{~Hz}), 7.25-7.23$ $(2 \mathrm{H}, \mathrm{d}, J=8.8 \mathrm{~Hz}), 7.17-7.49(2 \mathrm{H}, \mathrm{m}), 7.10-7.04(6 \mathrm{H}, \mathrm{m})$, 6.85-6.82 (2 H, m), 6.64-6.60 (4 H, m), 6.19-6.18 (1 H, d, $J=2$ $\mathrm{Hz}), 6.18-6.17(1 \mathrm{H}, \mathrm{d}, J=2 \mathrm{~Hz}), 6.11-6.10(1 \mathrm{H}, \mathrm{d}, J=2 \mathrm{~Hz})$, 6.08-6.07 (1 H, d, $J=2 \mathrm{~Hz}), 4.49-4.47(1 \mathrm{H}, \mathrm{d}, J=9.2 \mathrm{~Hz})$,

4.191-4.168 (1 H, d, J = 9.2 Hz), $3.94(1 \mathrm{H}, \mathrm{s}), 3.84(3 \mathrm{H}, \mathrm{s}), 3.83(3 \mathrm{H}, \mathrm{s}), 3.77$ (4 H, m), $3.75(3 \mathrm{H}, \mathrm{s}), 3.71(3 \mathrm{H}, \mathrm{s}), 3.66(4 \mathrm{H}, \mathrm{m}), 3.62(3 \mathrm{H}, \mathrm{s}), 3.55(3 \mathrm{H}, \mathrm{s}), 3.29(1 \mathrm{H}, \mathrm{s}) ;{ }^{13} \mathrm{C}$ NMR $\delta 205.5,170.7,170.6,161.9,161.3,158.8,158.6,158.4,153.6,152.8,139.9$, $138.1,130.1,129.8,128.9,128.7,128.2,127.8,127.9,127.0,126.5,125.6,113.6,112.7$, 112.6, 107.7, 106.5, 97.9, 95.5, 94.4, 94.3, 93.6, 93.4, 92.7, 88.7, 83.6, 81.04, 80.7, 62.4, 57.6, 56.1, 55.9, 55.4, 55.3, 55.1, 54.5, 53.4, 52.2, $51.8 \mathrm{ppm} ; \mathrm{HRMS}\left(\mathrm{CI} / \mathrm{NH}_{3}\right) \mathrm{m} / \mathrm{z}$ calculated for $\mathrm{C}_{28} \mathrm{H}_{26} \mathrm{O}_{8} 490.1628$ found $491.1739(\mathrm{M}+\mathrm{H})$.
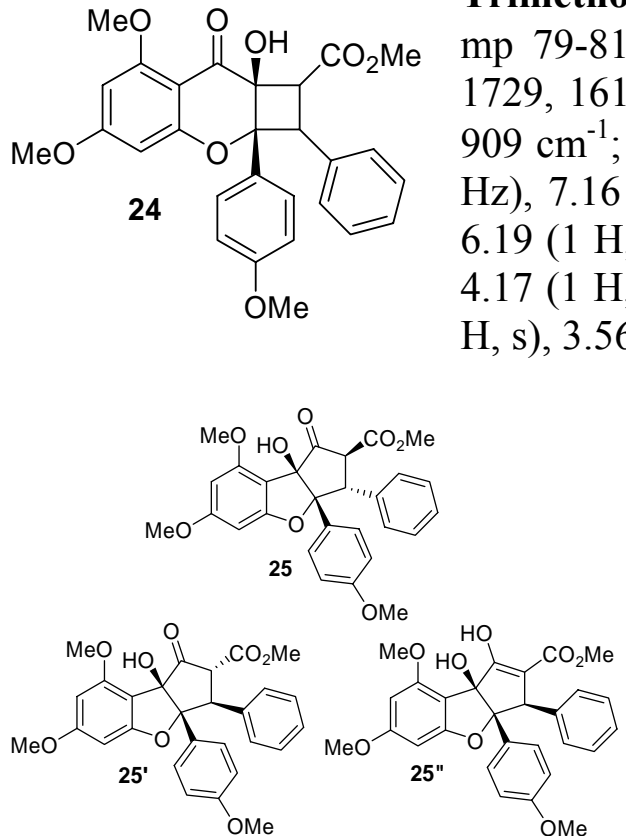

Trimethoxy benzo[b]cyclobutapyran-8-one 24. Yellow solid: mp 79-81 ${ }^{\circ} \mathrm{C}$. IR $v_{\max }$ (film): 3489, 3006, 2948, 2839, 1734, $1729,1618,1590,1516,1461,1437,1299,1200,1148,1096$, $909 \mathrm{~cm}^{-1} ;{ }^{1} \mathrm{H} \mathrm{NMR}\left(400 \mathrm{MHz}, \mathrm{CDCl}_{3}\right) \delta 7.53(2 \mathrm{H}, \mathrm{d}, J=8.8$ $\mathrm{Hz}), 7.16(2 \mathrm{H}, \mathrm{m}), 7.01(3 \mathrm{H}, \mathrm{m}), 6.64(2 \mathrm{H}, \mathrm{d}, J=8.8 \mathrm{~Hz})$, $6.19(1 \mathrm{H}, \mathrm{d}, J=2 \mathrm{~Hz}), 6.08(1 \mathrm{H}, \mathrm{d}, J=2 \mathrm{~Hz}), 4.27(1 \mathrm{H}, \mathrm{s})$, $4.17(1 \mathrm{H}, \mathrm{d}, J=9.6 \mathrm{~Hz}), 3.84(4 \mathrm{H}, \mathrm{m}), 3.75(3 \mathrm{H}, \mathrm{s}), 3.67$ (3 $\mathrm{H}, \mathrm{s}), 3.56(3 \mathrm{H}, \mathrm{s}) \mathrm{ppm}$

filtered, and concentrated in vacuo to afford $57 \mathrm{mg}(0.12 \mathrm{mmol}, 95 \%)$ of crude ketol shift product $\mathbf{2 5} / \mathbf{2 5} / \mathbf{2 5}$ ' as a yellow oil which was used without further purification $(3: 1$ mixture of endo:exo isomers $\mathbf{2 5} \mathbf{2 5}^{2}$ ':25). IR $v_{\max }$ (film): 3501, 3006, 2947, 2926, 2839, $1762,1734,1615,1513,1450,1440,1255,1213,1146,1033,1076 \mathrm{~cm}^{-1} ;{ }^{1} \mathrm{H}$ NMR (400 $\mathrm{MHz} \mathrm{CDCl}_{3}, 1: 1$ mixture of keto/enol tautomers 25':25') $\delta$ 7.34-7.32 (2 H, d, $J=6.8$ $\mathrm{Hz}), 7.20-7.19(2 \mathrm{H}, \mathrm{m}), 7.09-6.86(15 \mathrm{H}, \mathrm{m}), 6.65(2 \mathrm{H}, \mathrm{d}, J=8.8 \mathrm{~Hz}), 6.51$ (2 H., d, $J=$ $6.8 \mathrm{~Hz}), 6.33(1 \mathrm{H}, \mathrm{d}, J=1.6 \mathrm{~Hz}), 6.17(1 \mathrm{H}, \mathrm{d}, J=1.6 \mathrm{~Hz}), 6.13(1 \mathrm{H}, \mathrm{d}, J=1.6 \mathrm{~Hz})$, $6.12(1 \mathrm{H}, \mathrm{d}, J=1.6 \mathrm{~Hz}), 6.05(1 \mathrm{H}, \mathrm{d}, J=1.6 \mathrm{~Hz}), 6.00(1 \mathrm{H}, \mathrm{d}, J=1.6 \mathrm{~Hz}), 4.46(1 \mathrm{H}$, s), $4.42(1 \mathrm{H}, \mathrm{d}, J=14.8 \mathrm{~Hz}), 4.36(1 \mathrm{H}, \mathrm{d}, J=14.8 \mathrm{~Hz}), 4.22(1 \mathrm{H}, \mathrm{d}, \mathrm{J}=13.6 \mathrm{~Hz}), 4.04$ $(1 \mathrm{H}, \mathrm{d}, 13.6 \mathrm{~Hz}), 3.84(3 \mathrm{H}, \mathrm{s}), 3.08-3.79(9 \mathrm{H}, \mathrm{m}), 3.77(9 \mathrm{H}, \mathrm{m}), 3.70(6 \mathrm{H}, \mathrm{m}), 3.64(6$ $\mathrm{H}, \mathrm{m}), 3.57(3 \mathrm{H}, \mathrm{s}), 3.30(1 \mathrm{H}, \mathrm{s}), 3.01(1 \mathrm{H}, \mathrm{s}) \mathrm{ppm}$ HRMS (EI) m/z calculated for $\mathrm{C}_{28} \mathrm{H}_{26} \mathrm{O}_{8} 490.1628$ found $490.9634(\mathrm{M}+\mathrm{H})$.

Benzo[b]cyclobutapyran-8-one 24 was subjected to the aforementioned conditions using $20 \mathrm{mg}$ (0.041 mmol, 1 equiv) of $24 \mathrm{in} \mathrm{MeOH}(2 \mathrm{~mL})$ and $\mathrm{NaOMe}(5 \mathrm{mg}, 0.09 \mathrm{mmol}, 2.5$ equiv) in $\mathrm{MeOH}(1 \mathrm{~mL}) .18 \mathrm{mg}$ of crude ketol shift product $\mathbf{2 5} / \mathbf{2 5}$ " $(0.036,90 \%)$ was isolated and used without further purification (only endo isomer isolated). 
Hydroxyl-directed reduction of trimethoxy keto rocaglate 25. To a solution of $184 \mathrm{mg}$ ( $0.70 \mathrm{mmol}, 6$ equiv) of $\mathrm{Me}_{4} \mathrm{NBH}(\mathrm{OAc})_{3}$ and $63 \mu \mathrm{L}(1.16 \mathrm{mmol}, 10$ equiv) of acetic acid in $3 \mathrm{~mL}$ of $\mathrm{CH}_{3} \mathrm{CN}$ was added a solution of $57 \mathrm{mg}(0.12 \mathrm{mmol}, 1$ equiv) of 25 in $1 \mathrm{~mL}$ of $\mathrm{CH}_{3} \mathrm{CN}$. The resulting yellow solution was stirred for $12 \mathrm{~h}$ at $\mathrm{rt}$ before being quenched with $2 \mathrm{~mL}$ of sat. $\mathrm{NH}_{4} \mathrm{Cl}$. The solution was then treated with $1 \mathrm{~mL}$ of a $3 \mathrm{M}$ aqueous solution of sodium/potassium tartrate and stirred at $\mathrm{rt}$ for $30 \mathrm{~min}$. The aqueous solution was extracted with $\mathrm{CH}_{2} \mathrm{Cl}_{2}(2 \times 5 \mathrm{~mL})$. The combined organic layers were washed with brine, dried over $\mathrm{MgSO}_{4}$, filtered and concentrated in vacuo. Purification on silica gel (40 $\%$ EtOAc in hexane) afforded $30 \mathrm{mg}(0.030 \mathrm{mmol}, 51 \%)$ of the corresponding endo methyl rocaglate 26 and $18 \mathrm{mg}(0.017 \mathrm{mmol}, 27 \%)$ of the corresponding exo methyl rocaglate 27 .

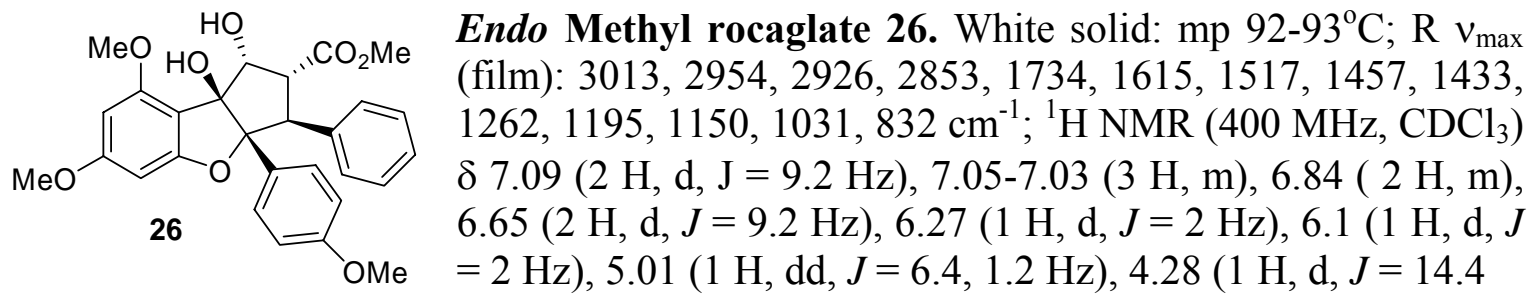
Hz), 3.80 (1 H, dd, $J=14.4,6.4 \mathrm{~Hz}), 3.86(3 \mathrm{H}, \mathrm{s}), 3.82(3 \mathrm{H}, \mathrm{s}), 3.69(3 \mathrm{H}, \mathrm{s}), 3.63(3 \mathrm{H}$, s), $3.50(1 \mathrm{H}, \mathrm{s}), 1.81(1 \mathrm{H}, \mathrm{br}) \mathrm{ppm} ;{ }^{13} \mathrm{C} \mathrm{NMR} \delta 170.5,164.1,160.9,158.8,157.0,137.0$, 129.0, 128.4, 127.8, 127.7, 126.5, 112.7, 107.7, 101.9, 93.7, 92.7, 89.5, 79.6, 60.4, 55.8, 55.1, 55.0, 51.9, $50.6 \delta \mathrm{HRMS}\left(\mathrm{CI} / \mathrm{NH}_{3}\right) \mathrm{m} / \mathrm{z}$ calculated for $\mathrm{C}_{28} \mathrm{H}_{28} \mathrm{O}_{8} 492.1784$ found $493.1891(\mathrm{M}+\mathrm{H})$.

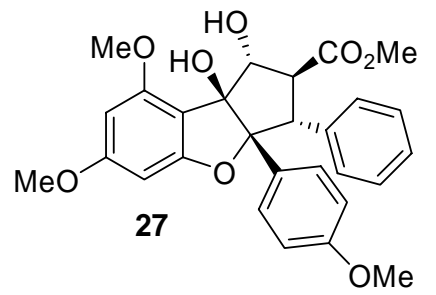

Exo Methyl rocaglate 27. Foamy yellow solid mp $84-85^{\circ} \mathrm{C}$. IR $v_{\max }$ (film): 3031, 3006, 2958, 2936, 2846, 1730, 1636, 1430, 1307, 1258, 1132, $103 \mathrm{~cm}^{-1} ;{ }^{1} \mathrm{H}$ NMR (400 MHz, $\left.\mathrm{CDCl}_{3}\right) \delta$ $7.34(2 \mathrm{H}, \mathrm{d}, J=8.8 \mathrm{~Hz}), 7.17-1.15(3 \mathrm{H}, \mathrm{m}), 6.95-6.94(2 \mathrm{H}$, $\mathrm{m}), 6.87(2 \mathrm{H}, \mathrm{d}, J=8.8 \mathrm{~Hz}), 6.12(1 \mathrm{H}, \mathrm{d}, J=1.6 \mathrm{~Hz}), 6.06(1$ $\mathrm{H}, \mathrm{d}, J=1.6 \mathrm{~Hz}), 4.76(1 \mathrm{H}, \mathrm{dd}, J=10.2,1.6 \mathrm{~Hz}), 4.02(1 \mathrm{H}, \mathrm{d}$, $J=12.8 \mathrm{~Hz}), 3.82(3 \mathrm{H}, \mathrm{s}), 3.78(3 \mathrm{H}, \mathrm{s}), 3.77(3 \mathrm{H}, \mathrm{s}), 3.60(3 \mathrm{H}, \mathrm{s}), 3.23(1 \mathrm{H}, \mathrm{dd}, J=$ 12.8, $10.2 \mathrm{~Hz}), 1.81(1 \mathrm{H}, \mathrm{s}) \mathrm{ppm} ;{ }^{13} \mathrm{C} \mathrm{NMR} \delta 173.1,164.1,162.0,159.4,157.9,135.0$, $129.1,128.4,128.0,127.3,119.7,113.6,105.1,99.5,92.6,91.4,88.8,83.9,55.8,55.8$, 55.4, 54.8, 52.3, 50.9; HRMS $\left(\mathrm{CI} / \mathrm{NH}_{3}\right) \mathrm{m} / \mathrm{z}$ calculated for $\mathrm{C}_{28} \mathrm{H}_{28} \mathrm{O}_{8} 492.1784$ found $493.1891(\mathrm{M}+\mathrm{H})$.

The crude ketol shift product $\mathbf{2 5}$ obtained from benzo[b]cyclobutapyran-8-one derivative 24 was subjected to the aforementioned conditions using, $58 \mathrm{mg}$ of $\mathrm{Me}_{4} \mathrm{NBH}(\mathrm{OAc})_{3}$ (0.22 mmol, 6 equiv), $20 \mu \mathrm{L}(0.37 \mathrm{mmol}, 10$ equiv) in $3 \mathrm{~mL}$ of $\mathrm{MeCN}$, and $18 \mathrm{mg}(0.037$ mmol, 1 equiv) of 25. $13 \mathrm{mg}$ of endo methyl rocaglate $26(0.021 \mathrm{mmol}, 75 \%)$ was obtained. 
Data comparison of natural ${ }^{\mathrm{S} 1}$ and synthetic endo methyl rocaglate

(26)<smiles>COc1ccc([C@]2(c3ccccc3)Oc3cc(OC)cc(OC)c3[C@@]2(O)[C@H](O)C(C)C)cc1</smiles>

Table 1. ${ }^{1} \mathrm{H}$ NMR Data for natural $\left(400 \mathrm{MHz}, \mathrm{CDCl}_{3}\right)$ and synthetic $\left(400 \mathrm{MHz}, \mathrm{CDCl}_{3}\right)$ endo methyl rocaglate 26.

\begin{tabular}{|c|c|c|}
\hline \multirow{2}{*}{ Pos. } & \multicolumn{2}{|c|}{${ }^{1} \mathrm{H}$ NMR $(\mathrm{Hz})$ in $\mathrm{CDCl}_{3}$} \\
\hline & Natural (400 MHz) & Synthetic $26(400 \mathrm{MHz})$ \\
\hline 1 & $5.02(\mathrm{dd}, 1.6,6.8)$ & $5.01(\mathrm{dd}, 1.2,6.4)$ \\
\hline $2 \beta$ & $3.91(\mathrm{dd}, 6.8,14.4)$ & $3.91(\mathrm{dd}, 6.4,14.4)$ \\
\hline $3 \alpha$ & $4.32(\mathrm{~d}, 14.4)$ & $4.27(\mathrm{~d}, 14.4)$ \\
\hline 5 & $6.29(\mathrm{~d}, 2.4)$ & $6.26(\mathrm{~d}, 2)$ \\
\hline 7 & $6.13(\mathrm{~d}, 2.4)$ & $6.10(\mathrm{~d}, 2)$ \\
\hline $2^{\prime}, 6^{\prime}$ & $7.11(\mathrm{~d}, 8.8)$ & $7.10(\mathrm{~d}, 9.2)$ \\
\hline $3^{\prime}, 5^{\prime}$ & $6.68(\mathrm{~d}, 8.8)$ & $6.65(\mathrm{~d}, 9.2)$ \\
\hline $2 ", 6 "$ & $6.88(\mathrm{~m})$ & $6.85(\mathrm{~m})$ \\
\hline $3 ", 4 ", 5 "$ & $7.07(\mathrm{~m})$ & $7.04(\mathrm{~m})$ \\
\hline OMe-6 & $3.88(\mathrm{~s})$ & $3.86(\mathrm{~s})$ \\
\hline OMe-8 & $3.84(\mathrm{~s})$ & $3.81(\mathrm{~s})$ \\
\hline OMe-4' & $3.71(\mathrm{~s})$ & $3.67(\mathrm{~s})$ \\
\hline $\mathrm{CO}_{2} \mathrm{Me}$ & $3.65(\mathrm{~s})$ & $3.62(\mathrm{~s})$ \\
\hline $\mathrm{OH}$ & $1.78,3.60(\mathrm{br}, \mathrm{s})$ & $1.88,3.50(\mathrm{br}, \mathrm{s})$ \\
\hline
\end{tabular}

Table 2. ${ }^{13} \mathrm{C}$ NMR Data for natural (75 MHz, acetone- $\left.d_{6}\right)$ and synthetic $\left(75 \mathrm{MHz}\right.$, acetone- $\left.d_{6}\right)$ endo methyl rocaglate 26.

\begin{tabular}{|c|c|c|}
\hline \multirow{2}{*}{ Pos. } & \multicolumn{2}{|c|}{${ }^{13} \mathrm{C} \mathrm{NMR}(\mathrm{Hz})$ in acetone $d_{6}$} \\
\hline & Natural (400 MHz) & Synthetic 26 (400 MHz) \\
\hline 1 & 80.6 & 80.3 \\
\hline 2 & 51.5 & 51.1 \\
\hline 3 & 55.8 & 55.5 \\
\hline $3 a$ & 102.6 & 102.2 \\
\hline 5 & 89.8 & 89.4 \\
\hline 7 & 92.8 & 92.3 \\
\hline $8 \mathrm{a}$ & 112.8 & 112.4 \\
\hline $8 \mathrm{~b}$ & 94.2 & 94.1 \\
\hline $1^{\prime}$ & 128.9 & 128.4 \\
\hline $2^{\prime}, 6{ }^{\prime}$ & 129.9 & 129.6 \\
\hline $3^{\prime}, 5^{\prime}$ & 112.8 & 112.4 \\
\hline $1 ”$ & 139.2 & 138.8 \\
\hline $2 ", 6 "$ & 128.2 & 128.4 \\
\hline $3 ", 5 "$ & 128.8 & 128.4 \\
\hline $4 "$ & 126.8 & 126.4 \\
\hline $4 \mathrm{a}, 6,8,4^{\prime}$ & $\begin{array}{c}158.6,159.3,161.7, \\
164.6\end{array}$ & $\begin{array}{c}158.3,158.9,161.4, \\
164.3\end{array}$ \\
\hline $\mathrm{ArOMe}$ & $55.2,55.9,56.0$ & $54.8,55.3,55.5$ \\
\hline $\mathrm{C}=\overline{\mathrm{O}}$ & 170.7 & 170.4 \\
\hline $\mathrm{CO}_{2} \mathrm{Me}$ & 51.5 & 51.1 \\
\hline
\end{tabular}

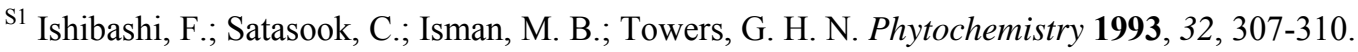
-S 8- 
Table 3. Miscellaneous data for natural and synthetic endo methyl rocaglate $\mathbf{2 6}$

\begin{tabular}{|l|l|l|}
\hline & Natural methyl rocaglate & Synthetic methyl rocaglate 26 \\
\hline Mp & $88-91$ & $92-93$ \\
\hline HRMS (EI), $m / z$ & $492.1797[M]^{+}$ & $492.1814[\mathrm{M}]^{+}$ \\
(rel. int.) & $492(3), 390(6), 313(46), 300$ & $492(2), 390(5), 313(40), 300$ \\
& $(100), 285(59), 181(66), 135$ & $(100), 285(23), 181(21), 135$ \\
& $(78), 131(50), 103(55)$. & $(16), 131(24)$, \\
\hline IR $v_{\max } \mathrm{cm}^{-1}(\mathrm{KBr})$ & $3489,1750,1623,1611,1513$, & $3486,1734,1615,1517,1251$, \\
& $1247,1218,1200,1149,1118$ & $1212,1195,1150,1115$. \\
\hline
\end{tabular}

\section{Data comparison of 27 and exo methyl rocaglate synthesized by Kraus and co-workers. ${ }^{\text {S2 }}$}

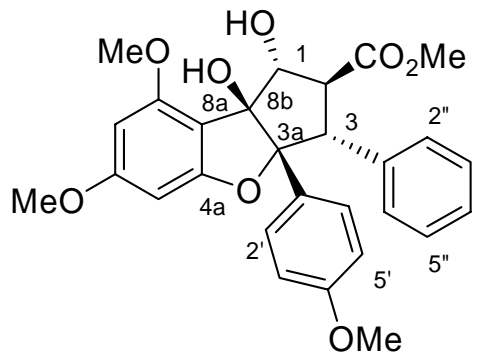

Table 4. ${ }^{1} \mathrm{H}$ NMR Data for Kraus' exo methyl rocaglate $(400 \mathrm{MHz}$, $\left.\mathrm{CDCl}_{3}\right)$ and compound $27\left(400 \mathrm{MHz}, \mathrm{CDCl}_{3}\right)$.

\begin{tabular}{|c|l|l|}
\hline \multirow{2}{*}{ Pos. } & \multicolumn{2}{|c|}{$\mathbf{H}$ NMR (Hz) in $\mathbf{C D C l}_{\mathbf{3}}$} \\
\cline { 2 - 3 } & $\begin{array}{c}\text { Exo methyl rocaglate (400 } \\
\mathbf{M H z})\end{array}$ & $\mathbf{2 7}(\mathbf{4 0 0} \mathbf{~ M H z})$ \\
\hline 1 & $4.77(\mathrm{~d}, 11)$ & $4.76(\mathrm{dd}, 1.6,10.2)$ \\
$2 \alpha$ & $3.24(\mathrm{dd}, 11,13)$ & $3.23(\mathrm{dd}, 10.2,12.8)$ \\
$3 \beta$ & $4.03(\mathrm{~d}, 13)$ & $4.02(\mathrm{~d}, 12.8)$ \\
5 & $6.12(\mathrm{~d}, 2)$ & $6.12(\mathrm{~d}, 1.6)$ \\
7 & $6.05(\mathrm{~d}, 2)$ & $6.06(\mathrm{~d}, 1.6)$ \\
$2,, 6$, & $7.33(\mathrm{~d}, 8)$ & $7.34(\mathrm{~d}, 8.8)$ \\
$3,, 5$, & $6.87(\mathrm{~d}, 8)$ & $6.87(\mathrm{~d}, 8.8)$ \\
$2 ", 6 "$ & $6.94(\mathrm{~m})$ & $7.16(\mathrm{~m})$ \\
$3 ", 4 ”, 5 "$ & $7.16(\mathrm{~m})$ & $3.82,3.78,3.77$ \\
$\mathrm{Ar}-\mathrm{OMe}$ & $3.81,3.78,3.76$ & 3.60 \\
$\mathrm{CO}_{2} \mathrm{Me}$ & 3.60 &
\end{tabular}

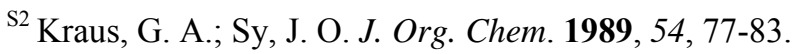

-S 9- 
Table 5. ${ }^{13} \mathrm{C}$ NMR Data for Kraus'exo methyl rocaglate $(75 \mathrm{MHz}$, $\left.\mathrm{CDCl}_{3}\right)$ and compound $27\left(75 \mathrm{MHz}, \mathrm{CDCl}_{3}\right)$.

\begin{tabular}{|c|c|c|}
\hline \multirow[b]{2}{*}{ Pos. } & \multicolumn{2}{|c|}{${ }^{13} \mathrm{C} \mathrm{NMR} \mathrm{(Hz)} \mathrm{in} \mathrm{CDCl}_{3}$} \\
\hline & $\begin{array}{c}\text { Exo methyl rocaglate (400 } \\
\text { MHz) (75 MHz) }\end{array}$ & 27 (75 MHz) \\
\hline 1 & 83.8 & 83.9 \\
\hline 2 & 50.8 & 50.90 \\
\hline 3 & 55.7 & 55.9 \\
\hline $3 a$ & 91.2 & 91.4 \\
\hline 5 & 88.7 & 88.7 \\
\hline 7 & 92.5 & 92.6 \\
\hline $8 \mathrm{a}$ & 105.0 & 105.1 \\
\hline $8 b$ & 99.3 & 99.5 \\
\hline 1 & 129.0 & 129.1 \\
\hline 2', 6' & missing & 119.6 \\
\hline $3^{\prime}, 5^{\prime}$ & 113.5 & 113.6 \\
\hline $1 "$ & 134.8 & 134.9 \\
\hline $2 ", 6 "$ & 128.3 & 128.4 \\
\hline $3 ", 5 "$ & 127.8 & 127.9 \\
\hline $4 "$ & 127.1 & 127.3 \\
\hline $4 a, 6,8,4$ & $\begin{array}{l}163.9,161.9,159.2, \\
156.8\end{array}$ & $\begin{array}{c}164.1,162.0,159.4, \\
157.9\end{array}$ \\
\hline $\mathrm{ArOMe}$ & $55.7,55.3,54.7$ & $55.8,55.4,54.8$ \\
\hline $\mathrm{C}=\overline{\mathrm{O}}$ & 172.95 & 173.1 \\
\hline $\mathrm{CO}_{2} \mathrm{Me}$ & 52.1 & 52.3 \\
\hline
\end{tabular}




\section{X-ray Crystal Structure of Compound 28:}

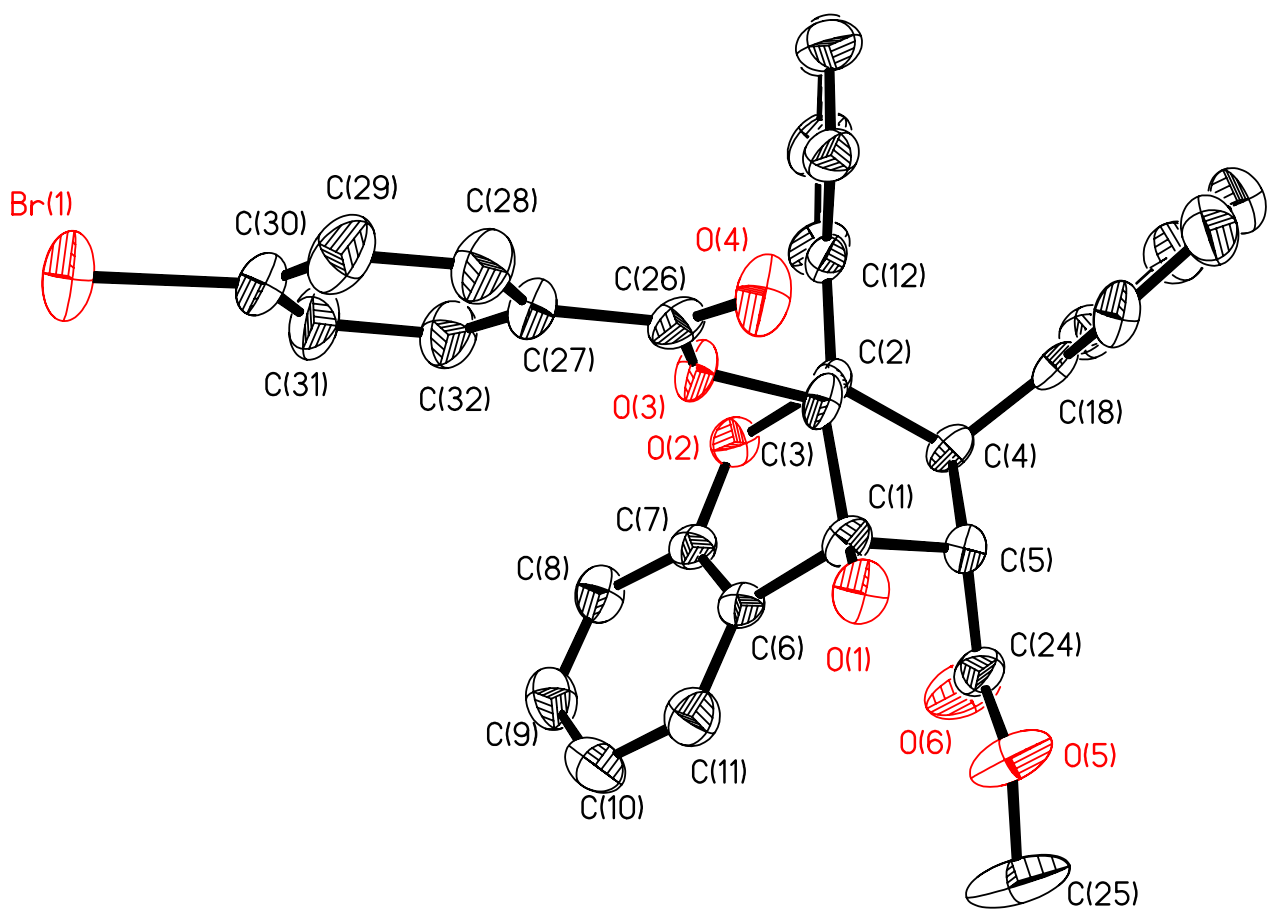

Crystals of compound $\mathbf{2 8}$ suitable for x-ray analysis were obtained by slow evaporation from benzene. Crystallographic data have been deposited with the Cambridge Crystallographic Data Centre (CCDC 248425). Copies of the data can be obtained free of charge on application to the CCDC, 12 Union Road, Cambridge CB21EZ, UK (fax: (+44)-1223-336-033; e-mail: deposit@ccdc.cam.ac.uk. 
Table 6. Crystal data and structure refinement for compound 28:

Identification code

Empirical formula

Formula weight

Temperature

Wavelength

Crystal system

Space group

Unit cell dimensions

Volume

Z

Density (calculated)

Absorption coefficient

$\mathrm{F}(000)$

Crystal size

Theta range for data collection

Index ranges

Reflections collected

Independent reflections

Completeness to theta $=25.00^{\circ}$

Absorption correction

Refinement method

Data / restraints / parameters

Goodness-of-fit on $\mathrm{F}^{2}$

Final $\mathrm{R}$ indices [I $>2 \operatorname{sigma}(\mathrm{I})]$

$\mathrm{R}$ indices (all data)

Largest diff. peak and hole
Compound 28

C50 H43 Br O6

819.75

213(2) K

$0.71073 \AA$

Monoclinic

P2(1)/c

$\mathrm{a}=12.027(2) \AA$

$\alpha=90^{\circ}$.

$\mathrm{b}=27.228(5) \AA$

$\beta=95.966(4)^{\circ}$.

$\mathrm{c}=12.927(2) \AA$

$\gamma=90^{\circ}$.

$4210.2(13) \AA^{3}$

$1.293 \mathrm{Mg} / \mathrm{m}^{3}$

$1.026 \mathrm{~mm}^{-1}$

1704

$0.10 \times 0.08 \times 0.08 \mathrm{~mm}^{3}$

1.70 to $25.00^{\circ}$.

$-14<=\mathrm{h}<=14,-32<=\mathrm{k}<=26,-12<=\mathrm{k}<=15$

22422

$7405[\mathrm{R}(\mathrm{int})=0.1260]$

$99.9 \%$

None

Full-matrix least-squares on $\mathrm{F}^{2}$

7405 / 0 / 516

0.998

$\mathrm{R} 1=0.0655, \mathrm{wR} 2=0.1101$

$\mathrm{R} 1=0.2038, \mathrm{wR} 2=0.1455$

0.504 and -0.513 e. $\AA^{-3}$ 
Table 7: Atomic coordinates $\left(x 1^{4}\right)$ and equivalent isotropic displacement parameters $\left(\AA^{2} \times 10^{3}\right)$ for compound 28 . $U(\mathrm{eq})$ is defined as one third of the trace of the orthogonalized $\mathrm{U}^{\mathrm{ij}}$ tensor.

\begin{tabular}{|c|c|c|c|c|}
\hline & $\mathrm{x}$ & $\mathrm{y}$ & $\mathrm{z}$ & $\mathrm{U}(\mathrm{eq})$ \\
\hline $\operatorname{Br}(1)$ & $7715(1)$ & $-21(1)$ & $11108(1)$ & $87(1)$ \\
\hline $\mathrm{O}(1)$ & $8382(3)$ & 544(1) & $4632(2)$ & $35(1)$ \\
\hline $\mathrm{O}(2)$ & $9728(3)$ & 1771(1) & $6227(2)$ & $32(1)$ \\
\hline $\mathrm{O}(3)$ & $9674(2)$ & $788(1)$ & $6723(2)$ & $31(1)$ \\
\hline $\mathrm{O}(4)$ & $10070(3)$ & $-13(1)$ & $6479(2)$ & $45(1)$ \\
\hline $\mathrm{O}(5)$ & 7898(3) & $1279(1)$ & 2796(3) & $56(1)$ \\
\hline $\mathrm{O}(6)$ & $8586(3)$ & $2004(2)$ & $3340(3)$ & $57(1)$ \\
\hline $\mathrm{C}(1)$ & $8941(4)$ & $988(2)$ & $4925(3)$ & $29(1)$ \\
\hline $\mathrm{C}(2)$ & 9981(4) & $923(2)$ & $5706(3)$ & $27(1)$ \\
\hline $\mathrm{C}(3)$ & $10475(4)$ & $1436(2)$ & $5747(3)$ & $26(1)$ \\
\hline $\mathrm{C}(4)$ & $10452(4)$ & $1565(2)$ & $4560(3)$ & $26(1)$ \\
\hline$C(5)$ & $9473(4)$ & $1255(2)$ & $4024(3)$ & $29(1)$ \\
\hline$C(6)$ & $8138(4)$ & $1319(2)$ & $5416(3)$ & $25(1)$ \\
\hline$C(7)$ & $8600(4)$ & $1700(2)$ & $6055(3)$ & $29(1)$ \\
\hline $\mathrm{C}(8)$ & $7922(4)$ & $2003(2)$ & $6570(3)$ & $36(1)$ \\
\hline $\mathrm{C}(9)$ & 6791(5) & $1937(2)$ & $6443(4)$ & $43(2)$ \\
\hline$C(10)$ & $6306(4)$ & $1570(2)$ & $5789(4)$ & $44(2)$ \\
\hline$C(11)$ & $7000(4)$ & $1269(2)$ & $5286(4)$ & $40(1)$ \\
\hline$C(12)$ & $11598(4)$ & $1516(2)$ & $6360(3)$ & $31(1)$ \\
\hline$C(13)$ & $12271(4)$ & $1133(2)$ & $6754(4)$ & $42(1)$ \\
\hline$C(14)$ & $13311(5)$ & $1225(3)$ & $7286(4)$ & $58(2)$ \\
\hline$C(15)$ & $13684(5)$ & 1704(3) & $7407(4)$ & $56(2)$ \\
\hline$C(16)$ & $13024(5)$ & $2086(2)$ & $7021(4)$ & $56(2)$ \\
\hline$C(17)$ & 11981(4) & $1997(2)$ & $6511(4)$ & $42(1)$ \\
\hline $\mathrm{C}(18)$ & $11563(4)$ & $1473(2)$ & 4139(3) & $30(1)$ \\
\hline C(19) & $11946(4)$ & $1004(2)$ & $3945(3)$ & $42(2)$ \\
\hline$C(20)$ & $12997(5)$ & $933(2)$ & $3630(4)$ & $56(2)$ \\
\hline $\mathrm{C}(21)$ & $13690(5)$ & $1318(3)$ & $3507(4)$ & $65(2)$ \\
\hline $\mathrm{C}(22)$ & $13332(5)$ & $1788(3)$ & $3692(4)$ & $60(2)$ \\
\hline $\mathrm{C}(23)$ & $12272(4)$ & $1864(2)$ & $4001(4)$ & $46(2)$ \\
\hline$C(24)$ & $8623(4)$ & $1569(2)$ & $3354(4)$ & $36(1)$ \\
\hline$C(25)$ & $6985(5)$ & $1530(2)$ & $2178(5)$ & $83(2)$ \\
\hline$C(26)$ & $9723(4)$ & 311(2) & 6993(4) & $31(1)$ \\
\hline$C(27)$ & $9263(4)$ & $242(2)$ & $8015(3)$ & $31(1)$ \\
\hline$C(28)$ & $9163(4)$ & $-227(2)$ & $8388(4)$ & $46(2)$ \\
\hline C(29) & $8711(4)$ & $-309(2)$ & $9317(4)$ & $54(2)$ \\
\hline$C(30)$ & $8390(4)$ & $85(3)$ & $9866(4)$ & $49(2)$ \\
\hline $\mathrm{C}(31)$ & $8494(4)$ & $557(2)$ & $9528(4)$ & $54(2)$ \\
\hline$C(32)$ & $8942(4)$ & $635(2)$ & $8579(4)$ & $44(1)$ \\
\hline $\mathrm{C}(1 \mathrm{~S})$ & $4769(8)$ & $1345(5)$ & $10026(8)$ & $93(3)$ \\
\hline $\mathrm{C}(2 \mathrm{~S})$ & $5621(12)$ & $1212(3)$ & $9503(8)$ & $99(3)$ \\
\hline$C(3 S)$ & $6349(9)$ & $1557(7)$ & $9179(6)$ & $121(5)$ \\
\hline$C(4 S)$ & $6183(10)$ & $2044(6)$ & $9438(10)$ & $126(4)$ \\
\hline$C(5 S)$ & $5309(14)$ & $2150(4)$ & $9966(10)$ & $132(5)$ \\
\hline$C(6 S)$ & $4636(9)$ & $1803(7)$ & $10255(7)$ & $124(4)$ \\
\hline$C(7 S)$ & $585(11)$ & $1764(4)$ & 1174(6) & 103(4) \\
\hline $\mathrm{C}(8 \mathrm{~S})$ & $1485(8)$ & 1631(3) & $723(10)$ & $100(3)$ \\
\hline $\mathrm{C}(9 \mathrm{~S})$ & $1469(8)$ & $1676(3)$ & $-344(10)$ & $98(3)$ \\
\hline $\mathrm{C}(10 \mathrm{~S})$ & $545(10)$ & $1846(3)$ & $-885(5)$ & $96(3)$ \\
\hline $\mathrm{C}(11 \mathrm{~S})$ & $-327(7)$ & 1983(3) & $-404(9)$ & $91(3)$ \\
\hline$C(12 S)$ & $-307(9)$ & 1932(3) & $610(10)$ & $100(3)$ \\
\hline$C(13 S)$ & 4997(6) & $9643(2)$ & $3812(5)$ & $66(2)$ \\
\hline $\mathrm{C}(14 \mathrm{~S})$ & $5880(5)$ & $9947(2)$ & $3817(4)$ & $59(2)$ \\
\hline$C(15 S)$ & $5985(5)$ & $10261(2)$ & $3000(5)$ & $57(2)$ \\
\hline$C(16 S)$ & $5181(6)$ & $10264(2)$ & $2162(5)$ & $67(2)$ \\
\hline $\mathrm{C}(17 \mathrm{~S})$ & $4263(5)$ & $9955(3)$ & $2147(5)$ & $74(2)$ \\
\hline $\mathrm{C}(18 \mathrm{~S})$ & $4184(5)$ & $9643(3)$ & $2990(6)$ & $74(2)$ \\
\hline
\end{tabular}




\section{X-ray Crystal Structure of Compound 29:}

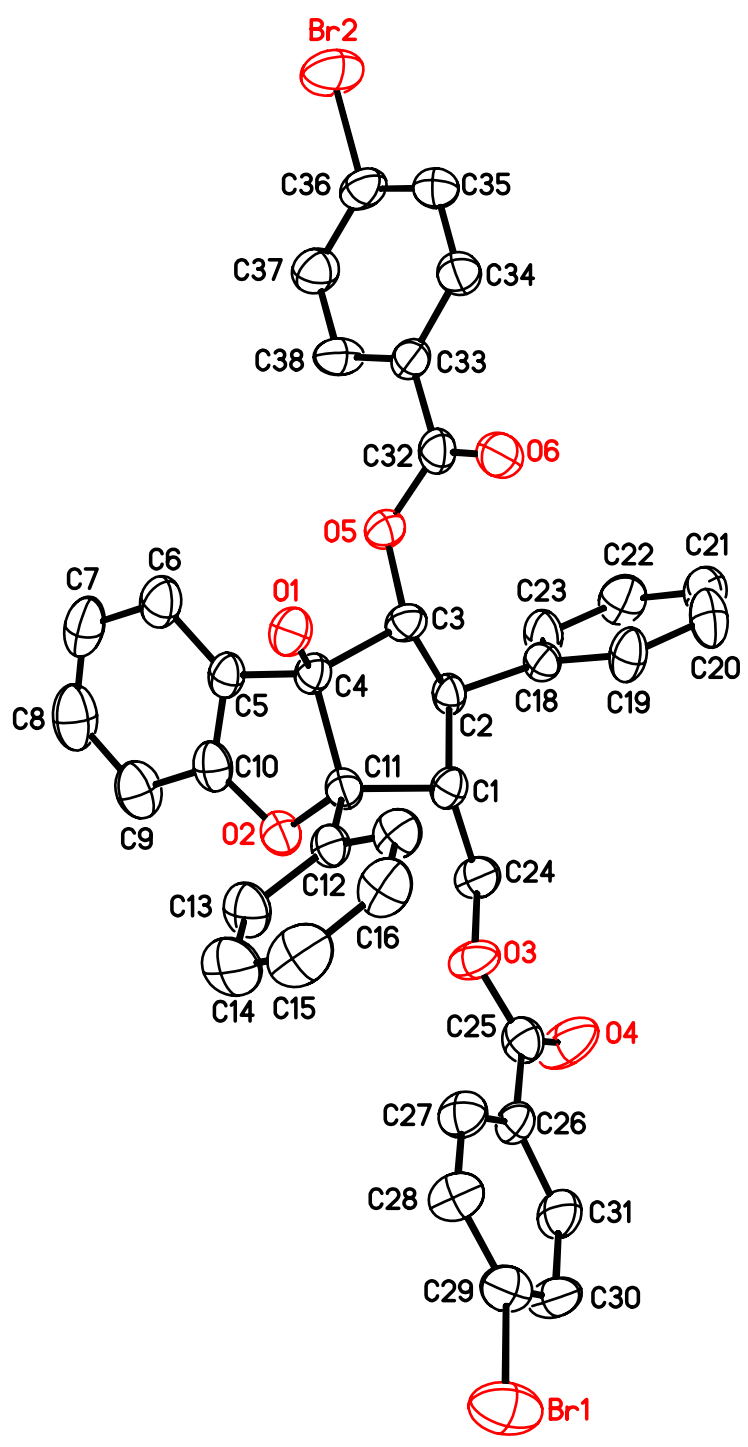

Crystals of 29 suitable for x-ray analysis were obtained by slow evaporation from benzene. Crystallographic data have been deposited with the Cambridge Crystallographic Data Centre (CCDC 248255). Copies of the data can be obtained free of charge on application to the CCDC, 12 Union Road, Cambridge CB21EZ, UK (fax: (+44)-1223336-033; e-mail: deposit@ccdc.cam.ac.uk. 
Table 8: Crystal data and structure refinement for compound 29.

Identification code

Empirical formula

Formula weight

Temperature

Wavelength

Crystal system

Space group

Unit cell dimensions

Volume

Z

Density (calculated)

Absorption coefficient

$\mathrm{F}(000)$

Crystal size

Theta range for data collection

Index ranges

Reflections collected

Independent reflections

Completeness to theta $=20.81^{\circ}$

Absorption correction

Max. and min. transmission

Refinement method

Data / restraints / parameters

Goodness-of-fit on $\mathrm{F}^{2}$

Final $\mathrm{R}$ indices [I $>2 \operatorname{sigma}(\mathrm{I})]$

$\mathrm{R}$ indices (all data)

Largest diff. peak and hole
Compound 29

C38 H28 Br2 O6

740.42

295(2) K

$0.71073 \AA$

Monoclinic

$\mathrm{P} 2(1) / \mathrm{c}$

$\mathrm{a}=25.4111(10) \AA$

$\alpha=90^{\circ}$.

$\mathrm{b}=16.5031(6) \AA$

$\beta=106.6770(10)^{\circ}$.

$\mathrm{c}=16.4599(6) \AA$

6612.3(4) $\AA^{3}$

8

$1.488 \mathrm{Mg} / \mathrm{m}^{3}$

$2.498 \mathrm{~mm}^{-1}$

2992

$0.40 \times 0.15 \times 0.03 \mathrm{~mm}^{3}$

0.84 to $20.81^{\circ}$.

$-25<=\mathrm{h}<=25,-13<=\mathrm{k}<=16,-14<=1<=16$

23839

$6644[\mathrm{R}(\mathrm{int})=0.0507]$

$95.9 \%$

Semiempirical by SADABS

0.9402 and 0.4348

Full-matrix least-squares on $\mathrm{F}^{2}$

6644 / 0 / 829

1.022

$\mathrm{R} 1=0.0459, \mathrm{wR} 2=0.1001$

$\mathrm{R} 1=0.0940, \mathrm{wR} 2=0.1169$

0.385 and -0.467 e. $\AA^{-3}$ 
Table 9: Atomic coordinates $\left(\times 1^{4}\right)$ and equivalent isotropic displacement parameters $\left(\AA^{2} \times 10^{3}\right)$ for compound 29. $U(\mathrm{eq})$ is defined as one third of the trace of the orthogonalized $U^{i j}$ tensor.

\begin{tabular}{|c|c|c|c|c|}
\hline & $\mathrm{X}$ & $\mathrm{y}$ & $\mathrm{Z}$ & $\mathrm{U}(\mathrm{eq})$ \\
\hline $\operatorname{Br}(1)$ & $3184(1)$ & $5716(1)$ & $4057(1)$ & $104(1)$ \\
\hline $\operatorname{Br}(2)$ & $7663(1)$ & $-3480(1)$ & $8044(1)$ & $108(1)$ \\
\hline $\mathrm{O}(1)$ & $5400(2)$ & $805(2)$ & $7217(2)$ & $60(1)$ \\
\hline $\mathrm{O}(2)$ & $6029(2)$ & $2606(2)$ & $6781(3)$ & $59(1)$ \\
\hline $\mathrm{O}(3)$ & $5314(2)$ & $3098(3)$ & $4920(3)$ & $68(1)$ \\
\hline $\mathrm{O}(4)$ & $5493(2)$ & $3702(3)$ & $3852(3)$ & $102(2)$ \\
\hline $\mathrm{O}(5)$ & $6276(2)$ & $-9(3)$ & $6592(2)$ & $53(1)$ \\
\hline $\mathrm{O}(6)$ & $5655(2)$ & $-918(3)$ & $5871(3)$ & $75(1)$ \\
\hline $\mathrm{C}(1)$ & $5662(2)$ & 1839(3) & $5500(4)$ & $49(2)$ \\
\hline $\mathrm{C}(2)$ & $6111(2)$ & $1183(3)$ & $5661(4)$ & $48(2)$ \\
\hline $\mathrm{C}(3)$ & $5901(2)$ & $634(3)$ & $6231(4)$ & $49(2)$ \\
\hline $\mathrm{C}(4)$ & $5798(2)$ & $1188(4)$ & $6905(4)$ & $48(2)$ \\
\hline$C(5)$ & $6292(3)$ & $1477(5)$ & $7593(4)$ & $53(2)$ \\
\hline $\mathrm{C}(6)$ & $6608(3)$ & $1072(5)$ & $8304(6)$ & $74(2)$ \\
\hline$C(7)$ & $7006(3)$ & $1509(7)$ & $8890(5)$ & $88(2)$ \\
\hline $\mathrm{C}(8)$ & 7091(3) & $2309(7)$ & $8763(6)$ & $96(3)$ \\
\hline C(9) & $6773(3)$ & $2715(5)$ & $8055(6)$ & $84(2)$ \\
\hline$C(10)$ & $6377(3)$ & $2274(5)$ & $7491(5)$ & $60(2)$ \\
\hline $\mathrm{C}(11)$ & $5609(2)$ & $2012(3)$ & $6395(4)$ & $48(2)$ \\
\hline$C(12)$ & $5063(3)$ & $2330(4)$ & $6436(4)$ & $51(2)$ \\
\hline$C(13)$ & $5032(3)$ & $2960(4)$ & $6972(4)$ & $73(2)$ \\
\hline$C(14)$ & $4528(4)$ & $3251(5)$ & $7011(5)$ & $97(3)$ \\
\hline$C(15)$ & $4052(4)$ & $2892(6)$ & $6548(6)$ & $96(3)$ \\
\hline$C(16)$ & $4076(3)$ & $2258(5)$ & $6032(5)$ & $77(2)$ \\
\hline$C(17)$ & $4579(3)$ & 1981(4) & $5983(4)$ & $65(2)$ \\
\hline$C(18)$ & $6209(3)$ & 764(3) & $4906(4)$ & $49(2)$ \\
\hline C(19) & $5788(3)$ & $456(4)$ & $4254(5)$ & $69(2)$ \\
\hline$C(20)$ & $5896(3)$ & $22(5)$ & $3608(5)$ & $84(2)$ \\
\hline$C(21)$ & $6419(4)$ & $-105(4)$ & $3583(5)$ & $83(2)$ \\
\hline$C(22)$ & $6845(3)$ & $208(5)$ & $4221(6)$ & $81(2)$ \\
\hline$C(23)$ & $6734(3)$ & $646(4)$ & $4872(4)$ & $70(2)$ \\
\hline$C(24)$ & $5773(3)$ & $2561(4)$ & $5011(4)$ & $63(2)$ \\
\hline$C(25)$ & $5213(3)$ & $3645(4)$ & $4320(5)$ & $60(2)$ \\
\hline$C(26)$ & $4731(3)$ & $4151(4)$ & $4288(4)$ & $54(2)$ \\
\hline$C(27)$ & $4410(3)$ & $4030(4)$ & $4819(4)$ & $65(2)$ \\
\hline$C(28)$ & 3958(3) & $4498(4)$ & $4772(4)$ & $68(2)$ \\
\hline C(29) & $3827(3)$ & $5098(4)$ & $4173(5)$ & $66(2)$ \\
\hline$C(30)$ & $4147(3)$ & $5259(4)$ & $3662(5)$ & $73(2)$ \\
\hline$C(31)$ & $4598(3)$ & $4779(4)$ & $3712(4)$ & $71(2)$ \\
\hline$C(32)$ & $6095(3)$ & $-767(4)$ & $6353(4)$ & $57(2)$ \\
\hline C(33) & $6498(3)$ & $-1401(4)$ & $6756(4)$ & $53(2)$ \\
\hline$C(34)$ & $6369(3)$ & $-2195(5)$ & $6560(4)$ & $67(2)$ \\
\hline$C(35)$ & $6710(3)$ & $-2805(4)$ & $6929(5)$ & $70(2)$ \\
\hline$C(36)$ & $7191(3)$ & $-2621(5)$ & $7518(5)$ & $68(2)$ \\
\hline$C(37)$ & $7339(3)$ & $-1839(5)$ & $7730(4)$ & $75(2)$ \\
\hline $\mathrm{C}(38)$ & 6991(3) & $-1219(4)$ & $7341(5)$ & $69(2)$ \\
\hline $\operatorname{Br}\left(1^{\prime}\right)$ & $11802(1)$ & $-10533(1)$ & $10881(1)$ & $124(1)$ \\
\hline $\operatorname{Br}\left(2^{\prime}\right)$ & $7410(1)$ & $-1294(1)$ & $10386(1)$ & $119(1)$ \\
\hline $\mathrm{O}\left(1^{\prime}\right)$ & $9479(2)$ & $-5770(2)$ & $11874(2)$ & $58(1)$ \\
\hline $\mathrm{O}\left(2^{\prime}\right)$ & $8841(2)$ & $-7557(2)$ & $10859(3)$ & $67(1)$ \\
\hline $\mathrm{O}\left(3^{\prime}\right)$ & $9596(2)$ & $-8089(3)$ & $9687(3)$ & $83(2)$ \\
\hline $\mathrm{O}\left(4^{\prime}\right)$ & $9470(2)$ & $-8664(3)$ & $8445(4)$ & $94(2)$ \\
\hline $\mathrm{O}\left(5^{\prime}\right)$ & $8687(2)$ & $-4920(3)$ & $10414(2)$ & $53(1)$ \\
\hline $\mathrm{O}\left(6^{\prime}\right)$ & $9349(2)$ & $-4085(2)$ & $10250(3)$ & $74(1)$ \\
\hline$C\left(1^{\prime}\right)$ & $9259(2)$ & $-6827(3)$ & 9939(4) & $55(2)$ \\
\hline$C\left(2^{\prime}\right)$ & $8840(2)$ & $-6142(3)$ & $9658(4)$ & $46(2)$ \\
\hline$C\left(3^{\prime}\right)$ & $9038(2)$ & $-5601(4)$ & $10424(4)$ & $48(2)$ \\
\hline
\end{tabular}




\begin{tabular}{|c|c|c|c|c|}
\hline$C\left(4^{\prime}\right)$ & $9097(2)$ & $-6149(4)$ & $11185(4)$ & $49(2)$ \\
\hline$C\left(5^{\prime}\right)$ & $8569(2)$ & $-6389(4)$ & $11375(4)$ & $51(2)$ \\
\hline$C\left(6^{\prime}\right)$ & $8232(3)$ & $-5955(4)$ & 11731(4) & $71(2)$ \\
\hline$C\left(7^{\prime}\right)$ & $7782(3)$ & $-6361(7)$ & $11888(4)$ & $91(3)$ \\
\hline $\mathrm{C}\left(8^{\prime}\right)$ & $7685(4)$ & $-7164(7)$ & $11667(6)$ & $103(3)$ \\
\hline $\mathrm{C}\left(9^{\prime}\right)$ & $8026(3)$ & $-7582(5)$ & $11318(5)$ & $89(2)$ \\
\hline $\mathrm{C}\left(10^{\prime}\right)$ & $8461(3)$ & $-7188(5)$ & $11179(4)$ & $61(2)$ \\
\hline$C\left(11^{\prime}\right)$ & $9278(2)$ & $-6985(4)$ & $10871(4)$ & $51(2)$ \\
\hline$C\left(12^{\prime}\right)$ & $9808(3)$ & $-7319(4)$ & $11431(4)$ & $53(2)$ \\
\hline$C\left(13^{\prime}\right)$ & $9818(3)$ & $-7944(4)$ & $11979(4)$ & $73(2)$ \\
\hline$C\left(14^{\prime}\right)$ & $10317(4)$ & $-8231(5)$ & $12519(5)$ & 101(3) \\
\hline$C\left(15^{\prime}\right)$ & 10799(4) & $-7873(6)$ & $12490(5)$ & $100(3)$ \\
\hline$C\left(16^{\prime}\right)$ & 10794(3) & $-7239(5)$ & $11965(5)$ & $84(2)$ \\
\hline$C\left(17^{\prime}\right)$ & 10299(3) & $-6968(4)$ & $11440(4)$ & $65(2)$ \\
\hline$C\left(18^{\prime}\right)$ & 8798(3) & $-5751(3)$ & 8818(4) & $46(2)$ \\
\hline$C\left(19^{\prime}\right)$ & $9258(3)$ & $-5451(4)$ & $8631(5)$ & $64(2)$ \\
\hline$C\left(20^{\prime}\right)$ & $9212(3)$ & $-5051(4)$ & $7879(5)$ & $76(2)$ \\
\hline$C\left(21^{\prime}\right)$ & 8713(4) & $-4936(4)$ & $7308(5)$ & $76(2)$ \\
\hline $\mathrm{C}\left(22^{\prime}\right)$ & $8262(3)$ & $-5240(5)$ & 7472(5) & $78(2)$ \\
\hline$C\left(23^{\prime}\right)$ & $8303(3)$ & $-5649(4)$ & $8228(5)$ & $65(2)$ \\
\hline$C\left(24^{\prime}\right)$ & $9144(3)$ & $-7551(4)$ & 9359(4) & $72(2)$ \\
\hline$C\left(25^{\prime}\right)$ & $9728(3)$ & $-8605(4)$ & $9179(6)$ & $66(2)$ \\
\hline$C\left(26^{\prime}\right)$ & 10214(3) & $-9097(4)$ & 9591(5) & $61(2)$ \\
\hline$C\left(27^{\prime}\right)$ & $10511(3)$ & $-8950(4)$ & $10431(6)$ & $71(2)$ \\
\hline $\mathrm{C}\left(28^{\prime}\right)$ & 10973(3) & $-9377(5)$ & $10847(5)$ & $80(2)$ \\
\hline$C\left(29^{\prime}\right)$ & $11149(3)$ & $-9964(5)$ & $10386(7)$ & $80(2)$ \\
\hline $\mathrm{C}\left(30^{\prime}\right)$ & 10872(4) & $-10141(4)$ & 9572(6) & $84(2)$ \\
\hline$C\left(31^{\prime}\right)$ & 10393(3) & $-9693(5)$ & $9164(5)$ & $78(2)$ \\
\hline $\mathrm{C}\left(32^{\prime}\right)$ & 8894(3) & $-4189(4)$ & $10318(4)$ & $50(2)$ \\
\hline$C\left(33^{\prime}\right)$ & $8516(3)$ & $-3514(4)$ & $10335(4)$ & $52(2)$ \\
\hline$C\left(34^{\prime}\right)$ & $8682(3)$ & $-2736(4)$ & $10216(4)$ & $63(2)$ \\
\hline$C\left(35^{\prime}\right)$ & $8351(3)$ & $-2087(4)$ & $10225(4)$ & $76(2)$ \\
\hline $\mathrm{C}\left(36^{\prime}\right)$ & 7861(3) & $-2200(4)$ & 10364(4) & $74(2)$ \\
\hline$C\left(37^{\prime}\right)$ & $7678(3)$ & $-2964(5)$ & $10488(4)$ & $76(2)$ \\
\hline$C\left(38^{\prime}\right)$ & $8009(3)$ & $-3627(4)$ & $10475(4)$ & $63(2)$ \\
\hline
\end{tabular}


${ }^{1} \mathrm{H}$ NMR spectrum $\left(400 \mathrm{MHz}, \mathrm{CD}_{3} \mathrm{CN}\right)$ of 3-hydroxyflavone $6(25 \mathrm{mg}, 0.12 \mathrm{mmol}$, 1 equiv) and methyl cinnamate $13(95 \mathrm{mg}, 0.6 \mathrm{mmol}, 5$ equiv) after $2 \mathrm{~h}$ of irradiation (uranium filter, $\lambda>350 \mathrm{~nm}$ ).

A) ${ }^{1} \mathrm{H}$ NMR spectrum $\left(400 \mathrm{MHz}, \mathrm{CD}_{3} \mathrm{CN}\right)$ of purified cyclopenta[bc]benzopyran 15

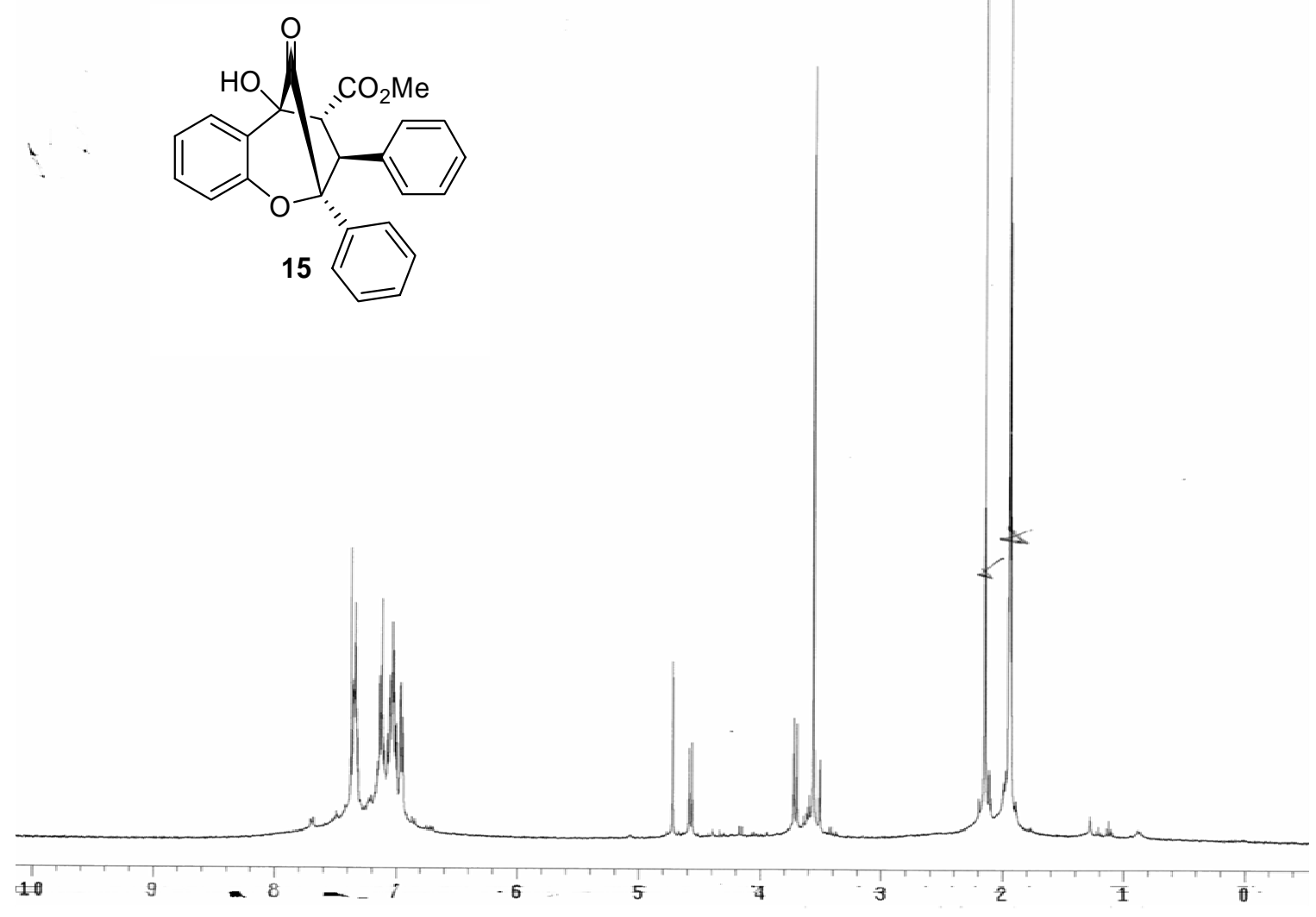

B) ${ }^{1} \mathrm{H}$ NMR spectrum (400 MHz, $\mathrm{CD}_{3} \mathrm{CN}$ ) of a mixture 3-hydroxyflavone (1 equiv) and methyl cinnamate (5 equiv) after $2 \mathrm{~h}$ of irradiation (uranium filter, $>350 \mathrm{~nm}$ )

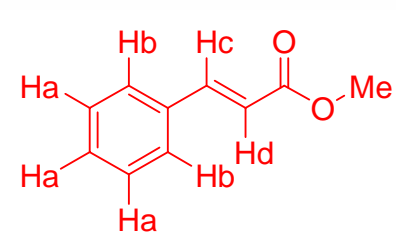

$\mathrm{OMe}$
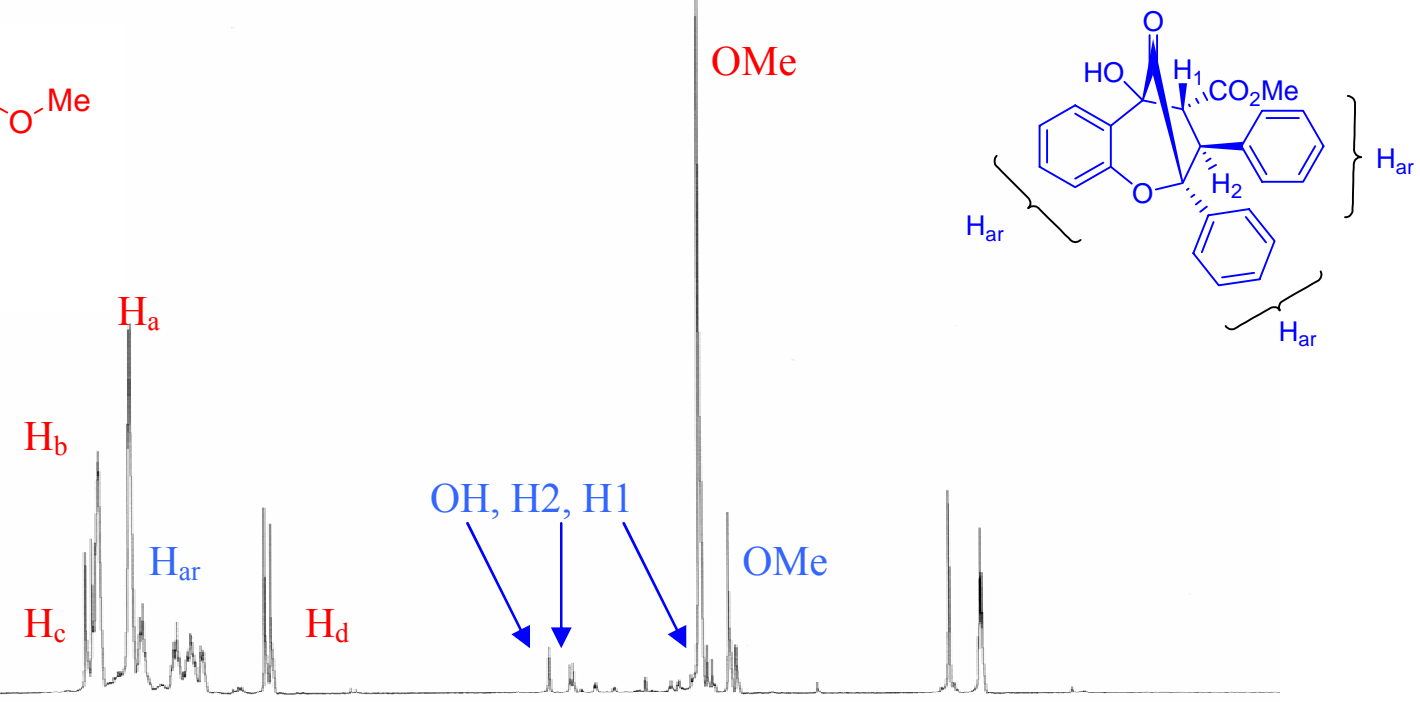
C) Expanded ${ }^{1} \mathrm{H}$ NMR spectrum (3 to $5 \mathrm{ppm}, 400 \mathrm{MHz}, \mathrm{CD}_{3} \mathrm{CN}$ ) of aglain 15 (left spectrum) and a mixture of 3-hydroxyflavone and methyl cinnamate after $2 \mathrm{~h}$ of irradiation (right spectrum).

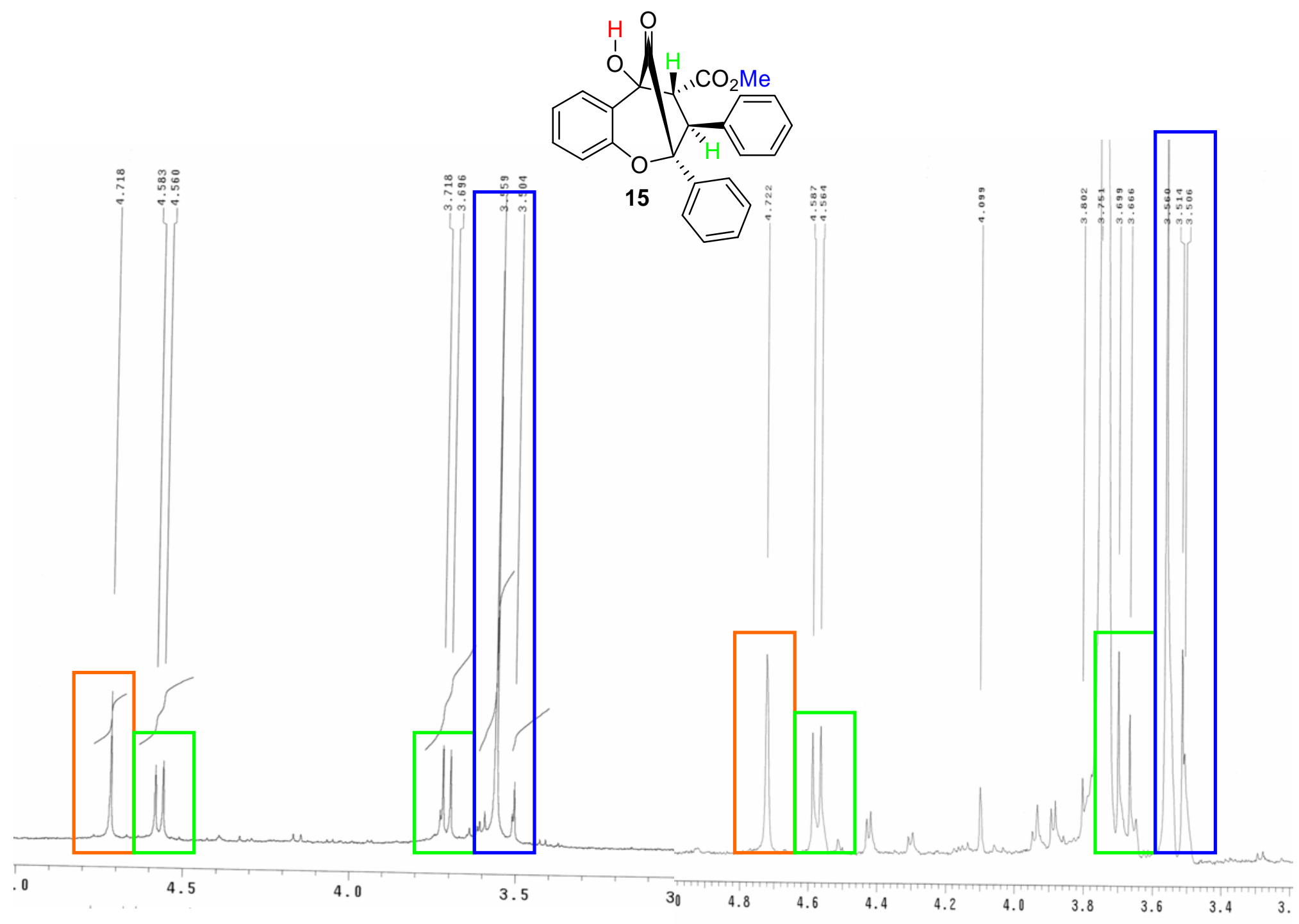




\section{${ }^{1} \mathrm{H}$ and ${ }^{13} \mathrm{C}$ NMR spectra for select compounds:}




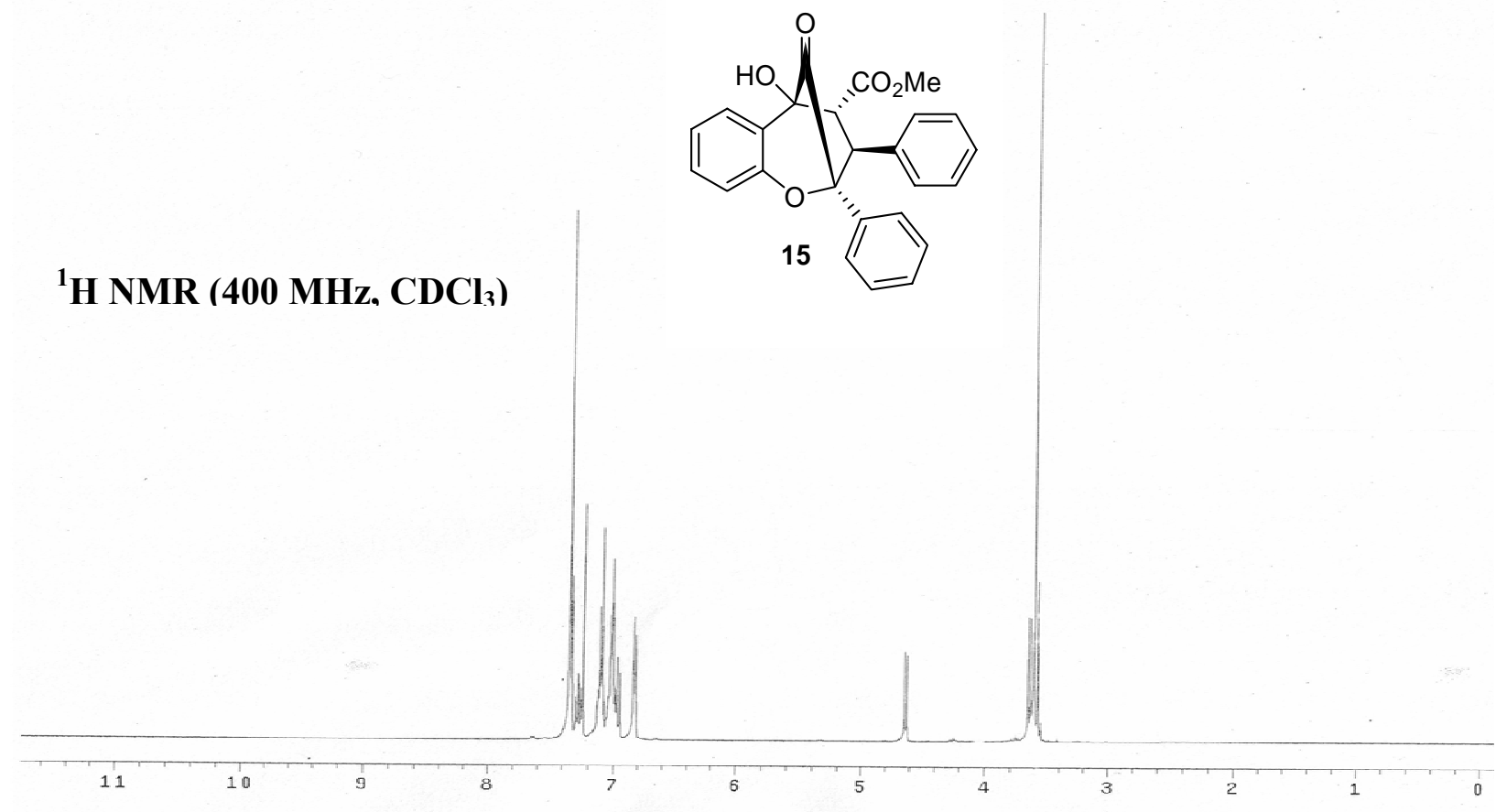

${ }^{13} \mathrm{C}$ NMR (75 MHz, $\mathrm{CDCl}_{3}$ )

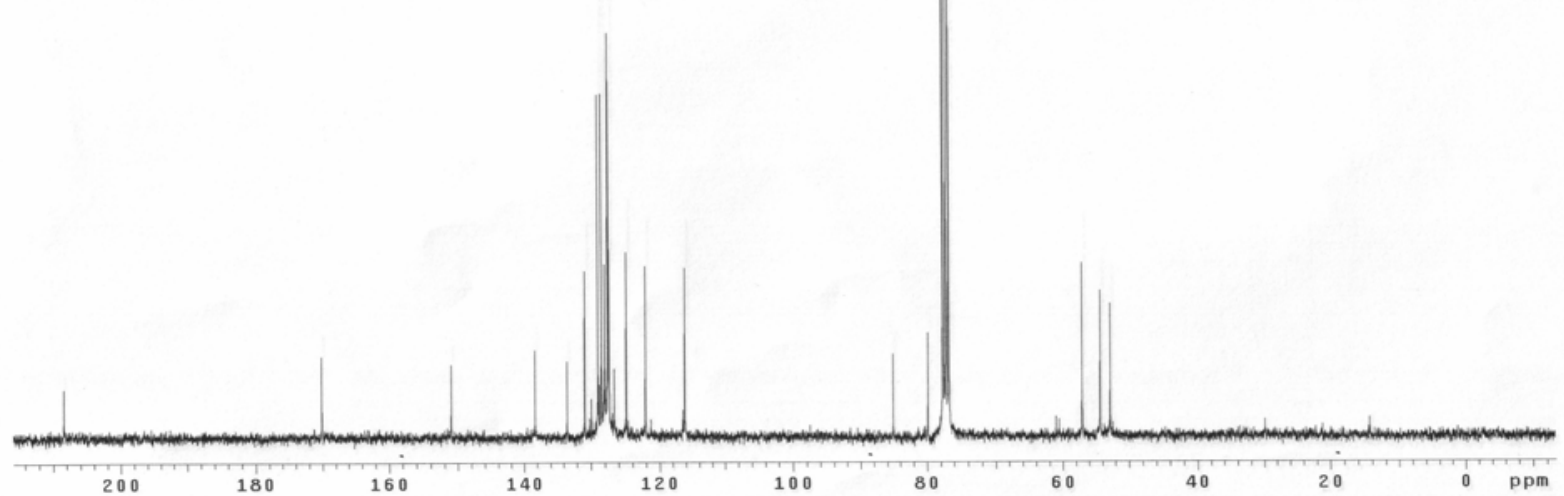

-S 21- 


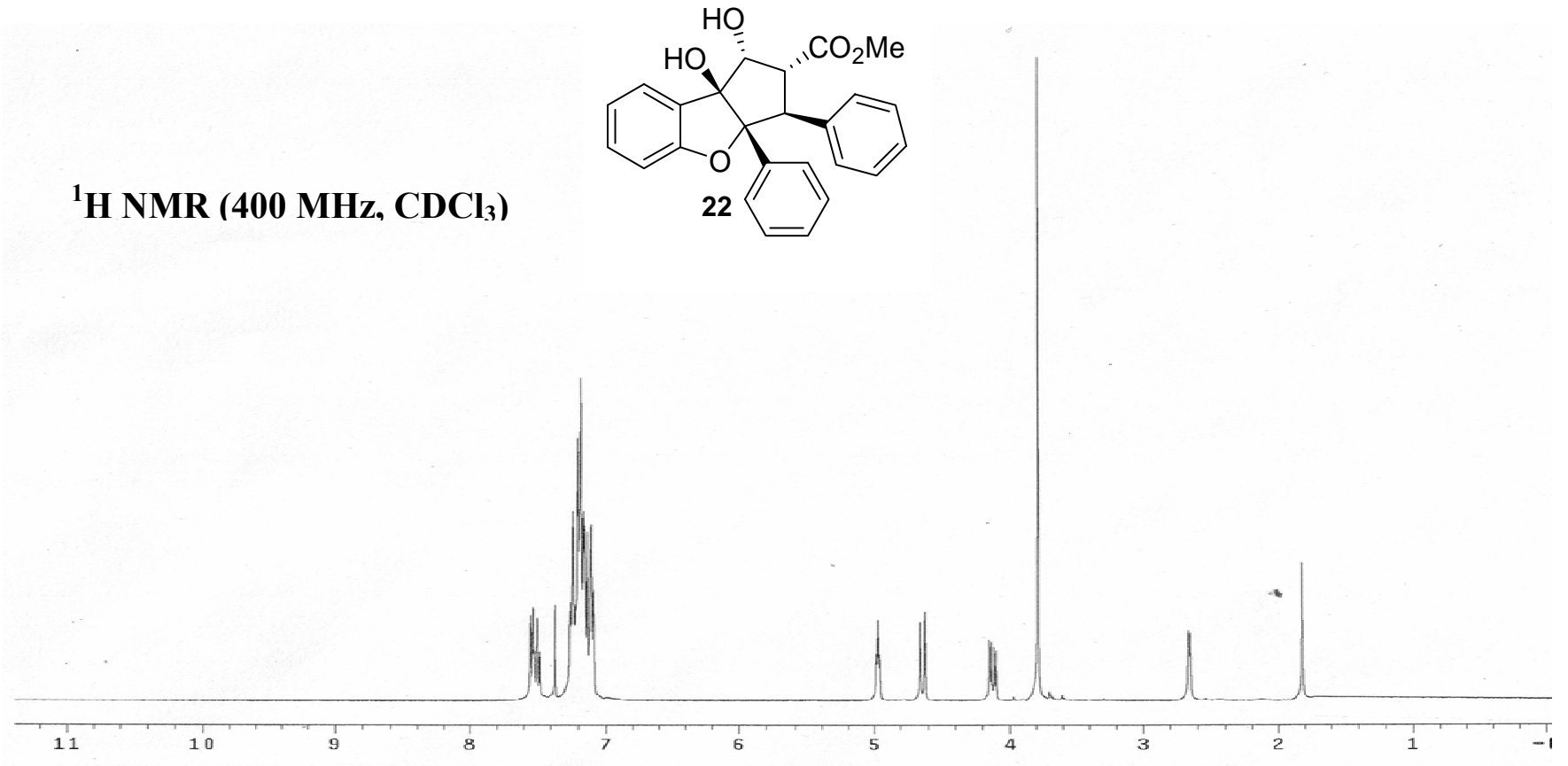

${ }^{13} \mathrm{C}$ NMR (75 MHz, $\mathrm{CDCl}_{3}$ )

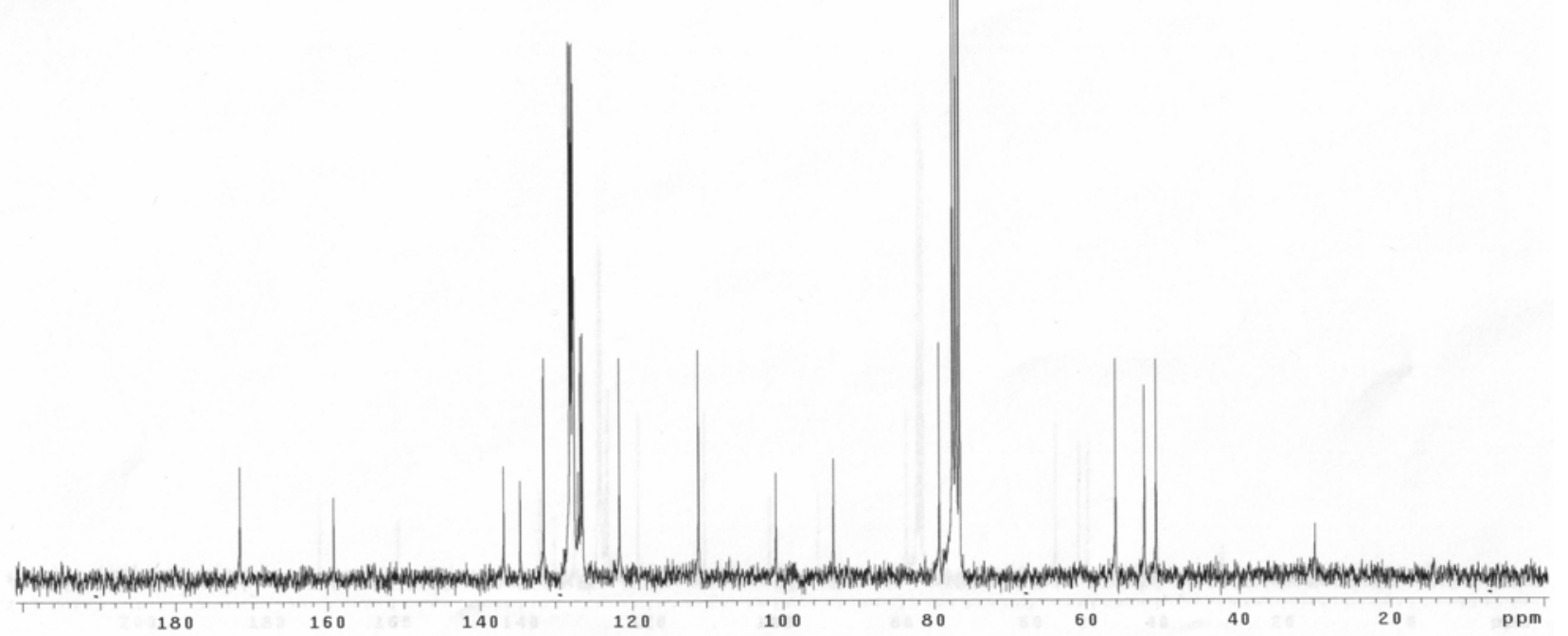




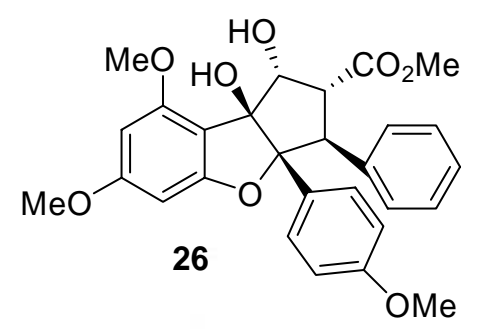

\section{${ }^{1} \mathrm{H}$ NMR (400 MHz, $\mathrm{CDCl}_{3}$ )}

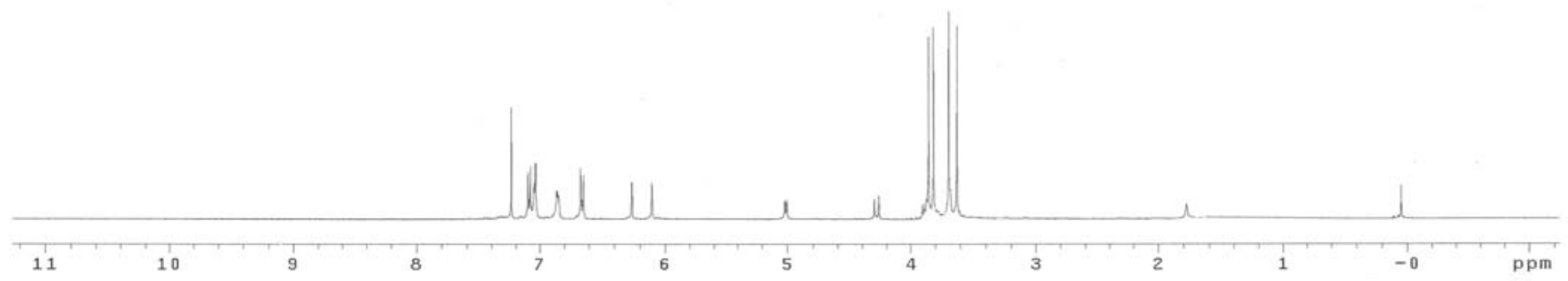

${ }^{13} \mathrm{C}$ NMR (75 MHz, $\mathrm{CDCl}_{3}$ )

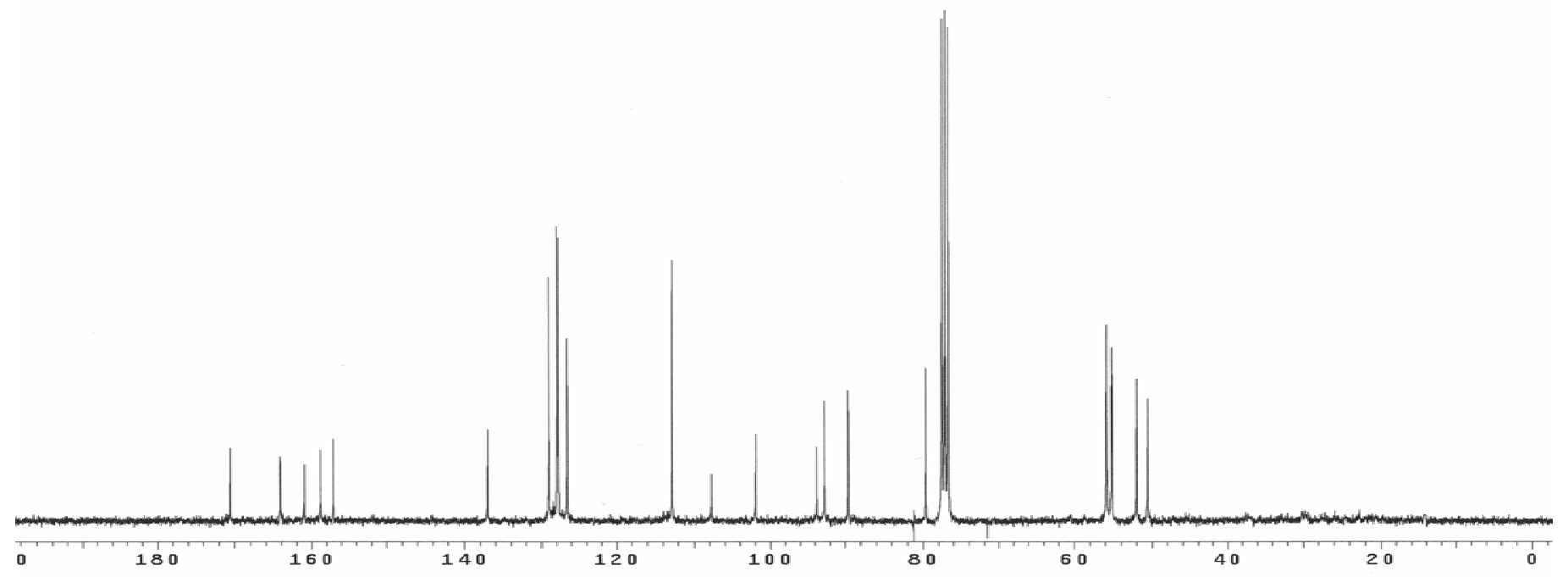

-S 23- 


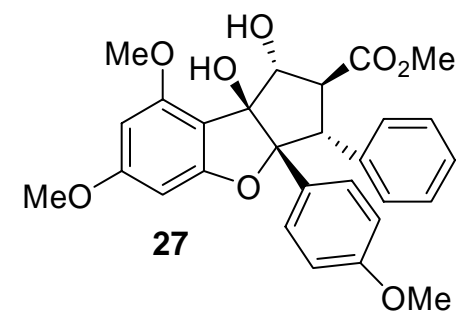

\section{${ }^{1}$ H NMR (400 MHz, $\left.\mathrm{CDCl}_{3}\right)$}

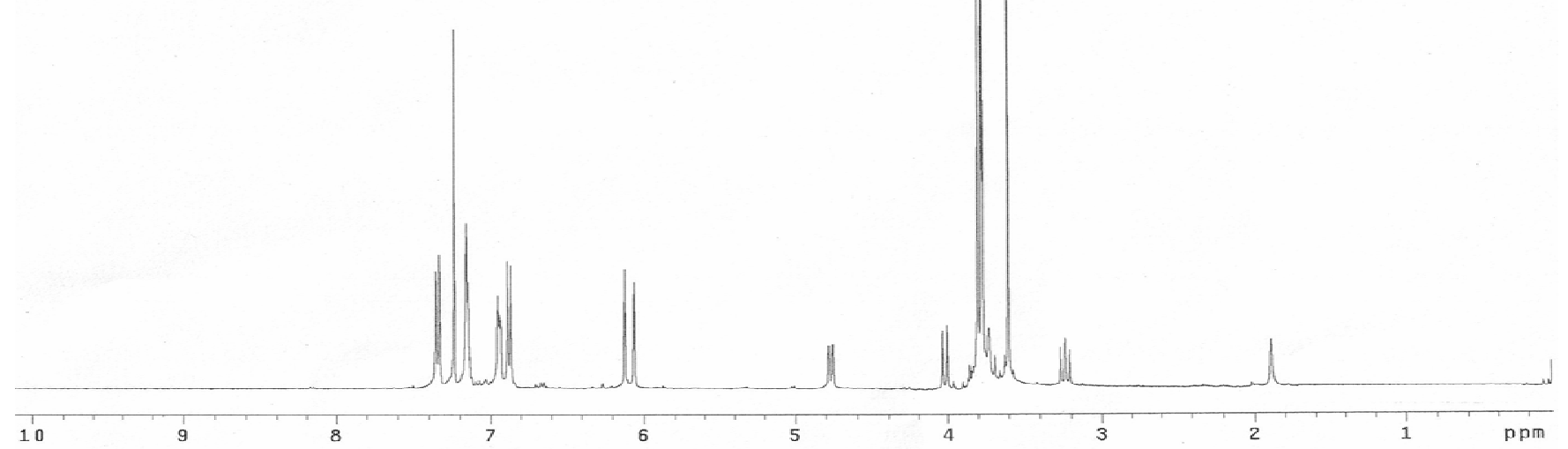

${ }^{13} \mathrm{C}$ NMR (75 MHz, $\left.\mathrm{CDCl}_{3}\right)$

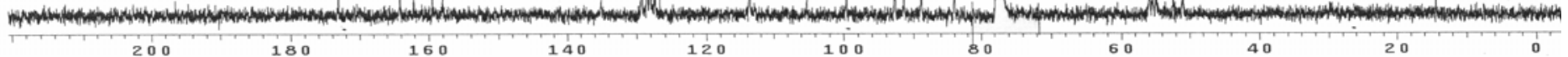


HMQC spectrum of synthetic exo methyl rocaglate $27\left(500 \mathrm{MHz}, \mathrm{CDCl}_{3}, 25{ }^{\circ} \mathrm{C}\right)$

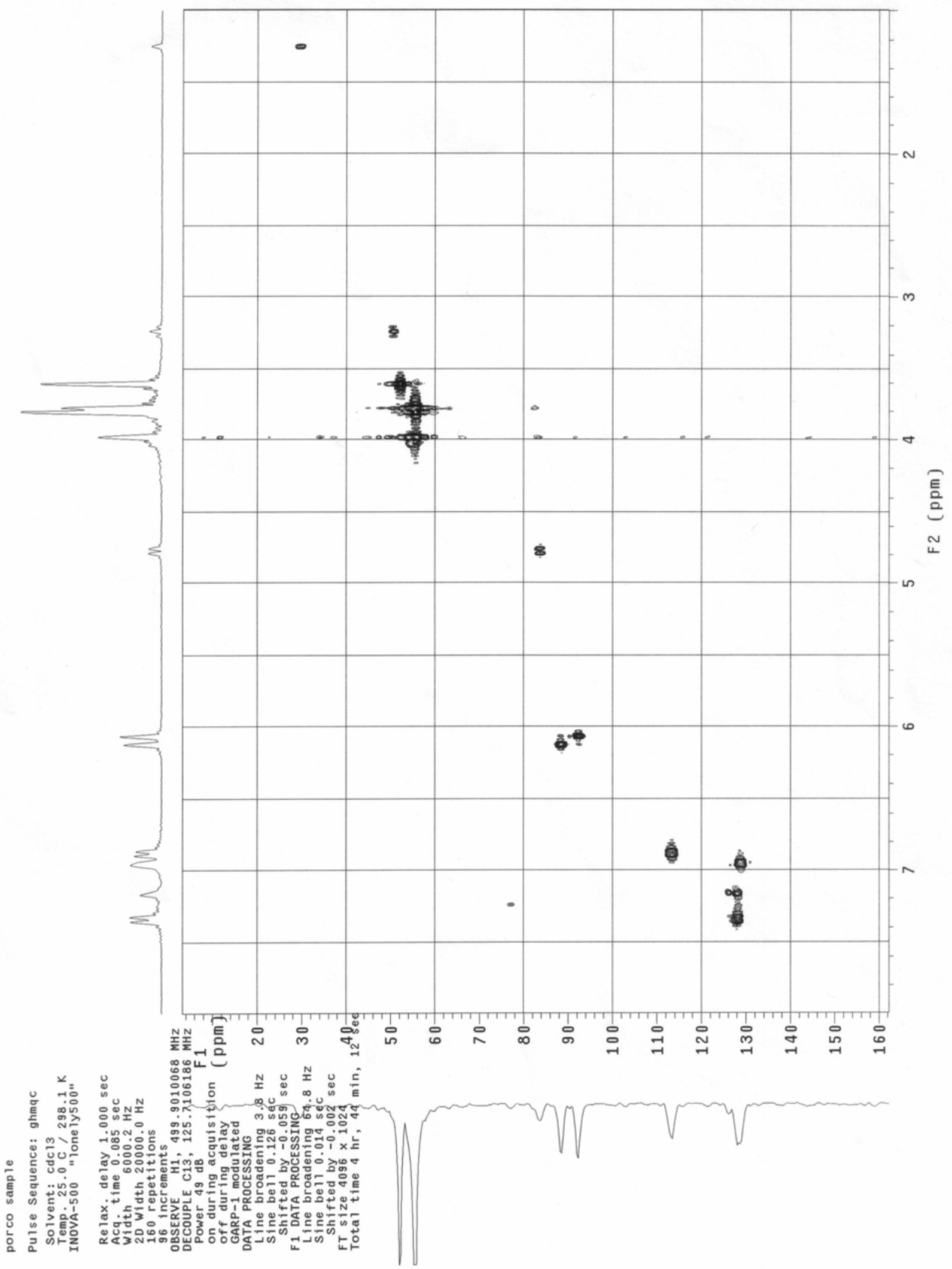


HMBC spectrum of synthetic exo methyl rocaglate $27\left(500 \mathrm{MHz}, \mathrm{CDCl}_{3}, 25^{\circ} \mathrm{C}\right)$

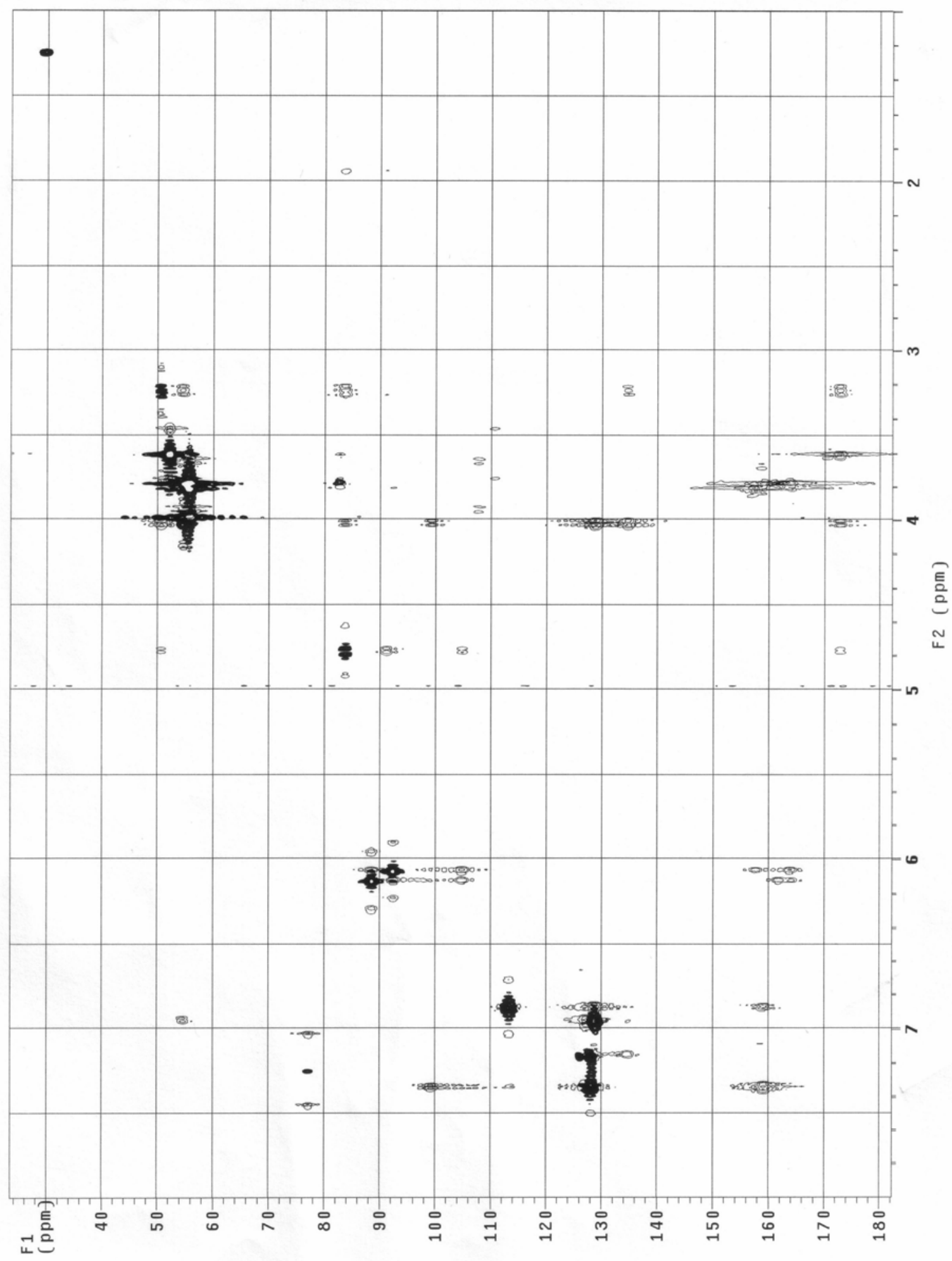


HMBC spectrum of synthetic methyl rocaglate $27\left(500 \mathrm{MHz}, \mathrm{CDCl}_{3} 25^{\circ} \mathrm{C}\right)$

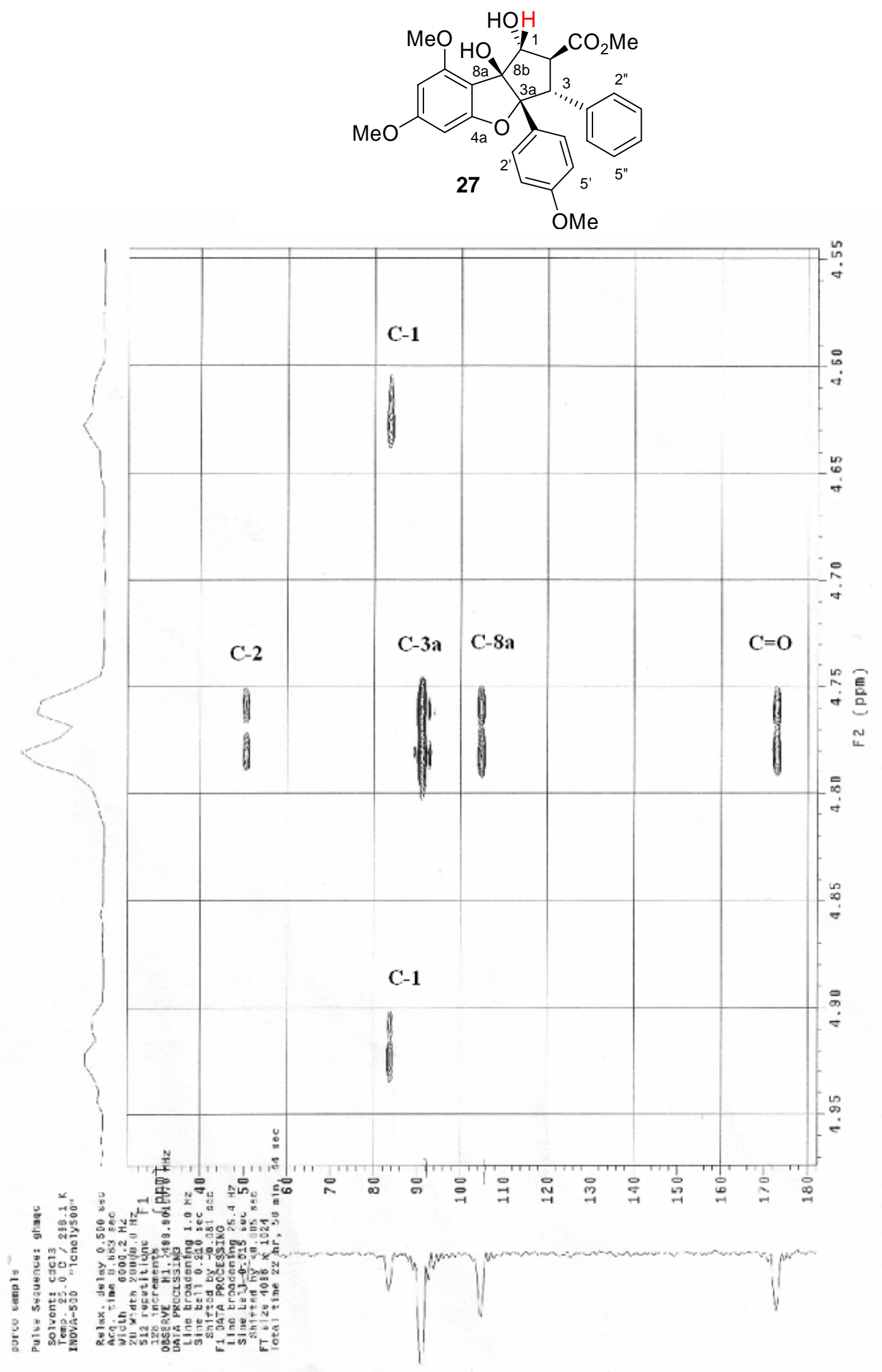

